\title{
Microstructure Distribution and Tensile Anisotropy of Dissimilar Friction Stir Welded AM60 and AZ31 Magnesium Alloys
}

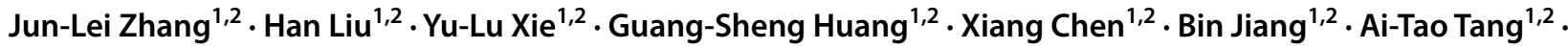 \\ Fu-Sheng Pan ${ }^{1,2}$
}

Received: 14 April 2020 / Revised: 5 May 2020 / Accepted: 11 May 2020 / Published online: 23 July 2020

(c) The Chinese Society for Metals (CSM) and Springer-Verlag GmbH Germany, part of Springer Nature 2020

\begin{abstract}
The microstructure distribution, tensile anisotropy and fracture behaviors in the dissimilar friction stir welded joint of AM60/ AZ31 alloys were investigated. Experimental results showed that a significant grain refinement and an orientation fluctuation occurred in the weld. The grain size of AZ31 side in joint was obviously smaller than that of AM60 side. There was a higher percentage of low angle grain boundaries (LAGBs) and a lower degree of recrystallization in AZ31 side compared with those in AM60 side, especially for the thermo-mechanically affected zone in AZ31 side. The discrepancies of grain size distribution, recrystallization behavior and LAGBs in joint depended on the different initial state of two metals and the inhomogeneous temperature distribution in joint. In addition, the (0001) basal plane in weld was roughly parallel to the surface of the pin, showing the symmetrically distributed texture characteristics. The joint showed an obvious tensile anisotropy due to the special texture distribution. The comprehensive tensile properties of joint along the three directions decreased in the order: welding direction, $45^{\circ}$ direction and transverse direction. The maximum ultimate tensile strength, yield strength and elongation of the joint were $242 \mathrm{MPa}, 116 \mathrm{MPa}$ and $21.2 \%$, respectively. The fluctuations of grain size and texture in joint affected the fracture behavior of samples in the three directions.
\end{abstract}

Keywords Friction stir welding $\cdot$ Dissimilar joint $\cdot$ Texture $\cdot$ Anisotropy $\cdot$ Tensile properties

\section{Introduction}

Mg alloys are regarded as the most potential structural material in the future of automotive and aerospace fields due to their advantages such as high specific strength and low density [1-3]. However, few slip systems can be activated at room temperature because of the hexagonal closepacked structure of the Mg alloy [1, 4, 5], which leads to its poor formability. Fortunately, welding, as an indispensable

Available online at http://link.springer.com/journal/40195.

Guang-Sheng Huang

gshuang@cqu.edu.cn

$\triangle$ Bin Jiang

jiangbinrong@cqu.edu.cn

1 State Key Laboratory of Mechanical Transmission, College of Materials Science and Engineering, Chongqing University, Chongqing 400044, China

2 National Engineering Research Center for Mg Alloys, Chongqing University, Chongqing 400044, China manufacturing method in industrial production, can largely compensate for the poor formability of $\mathrm{Mg}$ alloys. Friction stir welding (FSW) is known as the second revolution in welding history after laser welding [6]. It can realize the welding of metals without melting and therefore effectively avoid the problems (such as the evaporation loss of alloying elements, coarse grains and large deformation) in contrast to the traditional fusion welding $[3,6,7]$. Therefore, researches on FSW of Mg alloys have been increasing in recent years.

In previous reports [3-12], the FSW of Mg alloys mainly focused on the single $\mathrm{Mg}$ alloys, and the microstructure, texture evolution and mechanical properties of joints were more fully investigated. However, in recent years, with the in-depth study of $\mathrm{Mg}$ alloys, more series of $\mathrm{Mg}$ alloys have been successively developed. Due to the difference in properties between $\mathrm{Mg}$ alloys, realizing the welding of dissimilar $\mathrm{Mg}$ alloys can meet the requirements for the service performance of workpiece in different working environment [3]. At present, there were few reports on FSW of dissimilar $\mathrm{Mg}$ alloys, and these reports mainly revolved around the influence of material locations and welding parameters on the 
mechanical properties of joints [13-15]. The discussion on the microstructure and texture evolution of dissimilar FSW joints of $\mathrm{Mg}$ alloys was remarkably rare. Moreover, previous studies $[3,6,7,9,11,12]$ demonstrated that a special texture distribution in the joint can be easily formed during the FSW. That is, the grain orientation in weld gradually changed from the hard orientation for the basal slip and extension twinning of nugget zone (NZ) center to the soft orientation of NZ side. Texture is one of the important factors determining the properties of Mg alloys [11]. This special texture distribution of FSW joints is different from the homogeneous $\{0001\}$ basal texture in Mg alloy sheets. Therefore, the tensile anisotropy of $\mathrm{Mg}$ alloy joints may be different from that of $\mathrm{Mg}$ alloy sheets. However, the testing of the mechanical properties of FSW Mg joints mainly focused on single direction in previous reports [11-17]. There were few tests and comparative analysis of the mechanical properties of joints in different directions. Therefore, it is of great engineering significance to carry out the research on the tensile anisotropy of the FSW Mg joints to selectively avoid the weakest direction of the mechanical properties of joints.

Dissimilar FSW Mg joints may possess more complex microstructure distribution, joint mechanical properties and fracture behaviors due to the differences in the chemical composition and initial microstructure of the two metals. Therefore, it is necessary to investigate the microstructure distribution and anisotropic mechanical properties of FSW joints of dissimilar Mg alloys. AM60 and AZ31 are two types of common commercial Mg alloys. AZ31 is costeffective with a good ductility and extrudability; however, the corrosion resistance and impact resistance of AZ31 are meager [3]. As a contrast, AM60 possesses better impact resistance, corrosion resistance and strength at ambient temperature [3]. Because of their different properties, it is possible to obtain a new kind of material based on FSW, which can meet the needs of $\mathrm{Mg}$ alloys in different working environment. In our previous work [3], the FSW weldability of AM60/AZ31 Mg alloy was studied. The microstructure and texture distributions in various regions of the joint were not further compared and analyzed. In addition, the tensile anisotropy of the joint and their fracture behaviors remained unclear. Therefore, in this work, microstructure and texture distributions in each region of the joint were analyzed by electron backscatter diffraction (EBSD). The mechanical properties of the joint in different tensile directions were tested, and the fracture mechanisms of them were discussed.

\section{Experimental}

The experimentally used $\mathrm{Mg}$ alloy sheets were the commercial extruded AZ31 and AM60 with a thickness of $4.4 \mathrm{~mm}$. The stirring tool used for welding was a steel tapered cylinder pin, and the detailed dimensions are shown in Fig. 1a. The sheets after mechanical descaling were welded under the welding parameters of $100 \mathrm{~mm} / \mathrm{min}$ in welding speed, $800 \mathrm{rpm}$ in rotation rate and $2.5^{\circ}$ in tilt angle. The welding direction was at an angle of $0^{\circ}$ with the extrusion direction (ED). Two kinds of material locations were used for FSW, one was called as AM60/AZ31 joint [with AZ31 as retreating side (RS) and AM60 as advancing side (AS)], and the other was AZ31/AM60 joint (with AM60 as RS and AZ31 as AS).

The transverse cross-sectional sample of the joint perpendicular to the welding direction (WD) was used for the observations of optical microscope (OM) and EBSD. The OM samples were first mechanically polished and then etched using a solution of $1 \mathrm{ml}$ glacial acetic acid, $7 \mathrm{ml}$ ethanol and $0.42 \mathrm{~g}$ picric acid. The samples used for EBSD characterization were electropolished in a commercial AC2 solution at $-20{ }^{\circ} \mathrm{C}$ with a direct current voltage of $20 \mathrm{~V}$ for $120 \mathrm{~s}$. The EBSD tests were performed on a JOEL JSM $7800 \mathrm{~F}$ field emission scanning electron microscope with $20 \mathrm{kV}$. The EBSD data were analyzed via a HKL EBSD system. The dog bone-shaped tensile specimens along the $0^{\circ}, 45^{\circ}$ and $90^{\circ}$ directions were cut from the joint, and these dimensions are shown in Fig. 1b. Tensile tests were carried out on a universal testing machine with a strain rate of $1 \times 10^{-3} \mathrm{~s}^{-1}$ at room temperature. Three tensile tests were performed on each type of tensile specimen to ensure the reliability of the experimental results. Tensile specimens after fracture were analyzed by scanning electron microscope (SEM), OM and EBSD.

\section{Results and Discussion}

\subsection{Microstructure Distribution}

Figure 2 presents the cross-sectional morphology of the FSW joints. The cross-sectional morphology showed that the defect-free FSW joint was obtained and four zones of NZ, thermo-mechanically affected zone (TMAZ), heat-affected zone (HAZ) and base material (BM) were recognizable. It can be seen that, whether AM60 or AZ31 on RS, the FSW joints presented good formability at the FSW parameters. This indicated that the two kinds of BMs had similar plastic deformation capability. Similar results were also demonstrated in the dissimilar FSW of AZ31 and ZK60 Mg alloys [14]. The NZ was marked by the white dash lines. The NZ displayed a morphology of "bowl like" since NZ consisted of the crown zone and stir zone. During the welding process, the upper metal was subjected to two forces from the shoulder and stirring pin, while the lower metal was primarily subjected to the rotational shear force from the stirring pin. This led to the different shapes of the two regions. The grains 


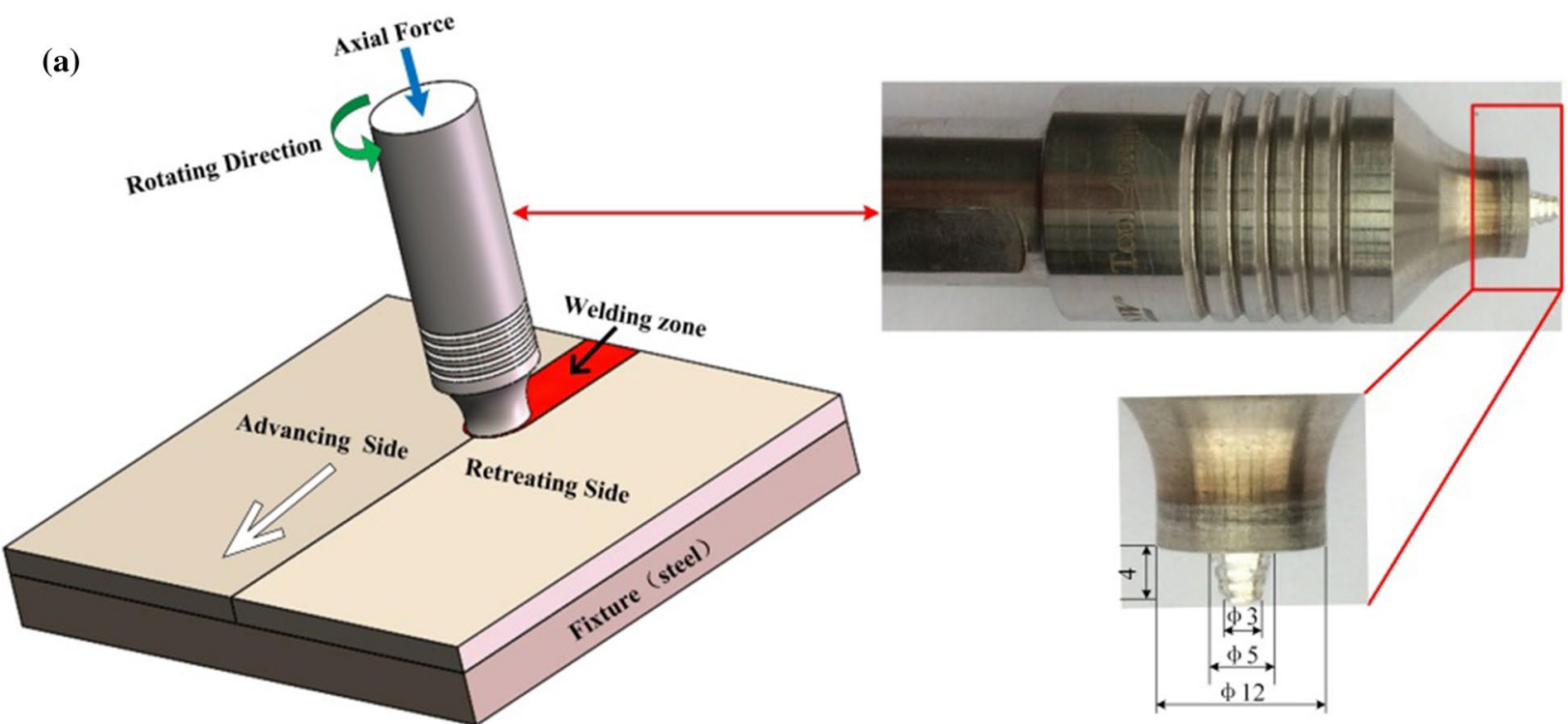

(b)

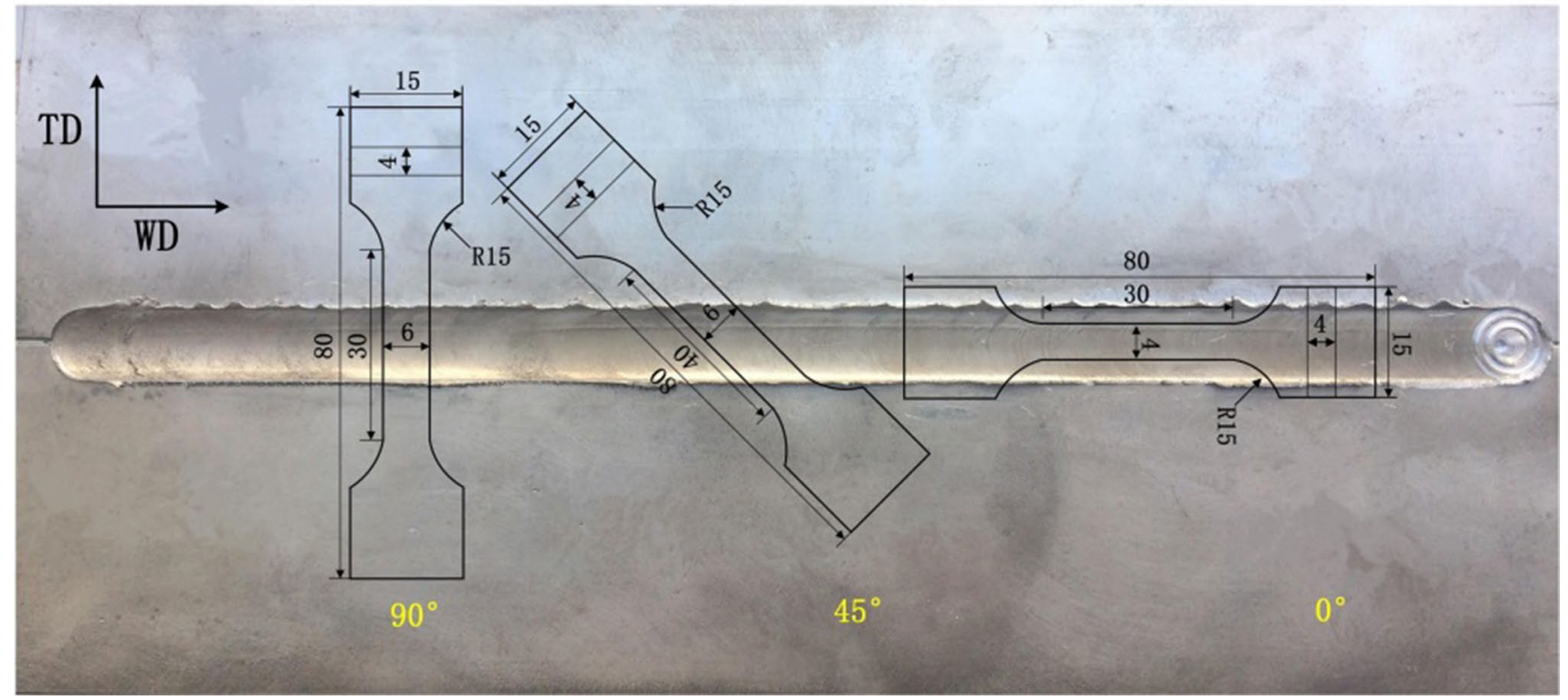

Fig. 1 Schematic illustration of FSW a illustration of sample preparation for tensile test $\mathbf{b}$

in NZ were refined obviously under the stirring action of the tool, which made the area distinct from the other regions of the joint. Furthermore, the interface between AZ31 and AM60 in NZ can also be visibly identified because of the discrepancy in grain size (Fig. 3) and corrosion resistance.

EBSD was operated at the corresponding locations of a-g points in Fig. 2a. Figure 3 shows the inverse pole figures (IPFs) of various regions of the joint. The colors in the IPF maps revealed the crystallographic orientation of each grain parallel to the normal direction (ND) of the sheets, according to the IPF color-coding shown in the figure. In Fig. 3, the main grain colors of the as-received AM60 and AZ31 BMs were red, implying that the grain orientations of both
BMs were mainly $<0001>$. This was the common feature of extruded Mg alloys sheets. Further, the microstructure of AM60 was primarily equiaxed recrystallized grains, and the average grain size was $\sim 14.7 \mu \mathrm{m}$ (Fig. 4a). However, the as-received AZ31 was an inhomogeneous microstructure, which showed a bimodal distribution. The average grain size was $\sim 4.8 \mu \mathrm{m}$ (Fig. $4 \mathrm{a}$ ). The bimodal microstructure of AZ31 BM could be caused by insufficient driving force for dynamic recrystallization during the hot deformation.

After FSW, the grain orientations in TMAZ changed significantly compared with that in BMs (Fig. 3). The grain orientation gradually transited from $<0001>$ in BMs to $<11-20>$ or $<10-10>$ in two TMAZs. The TMAZ exhibited 


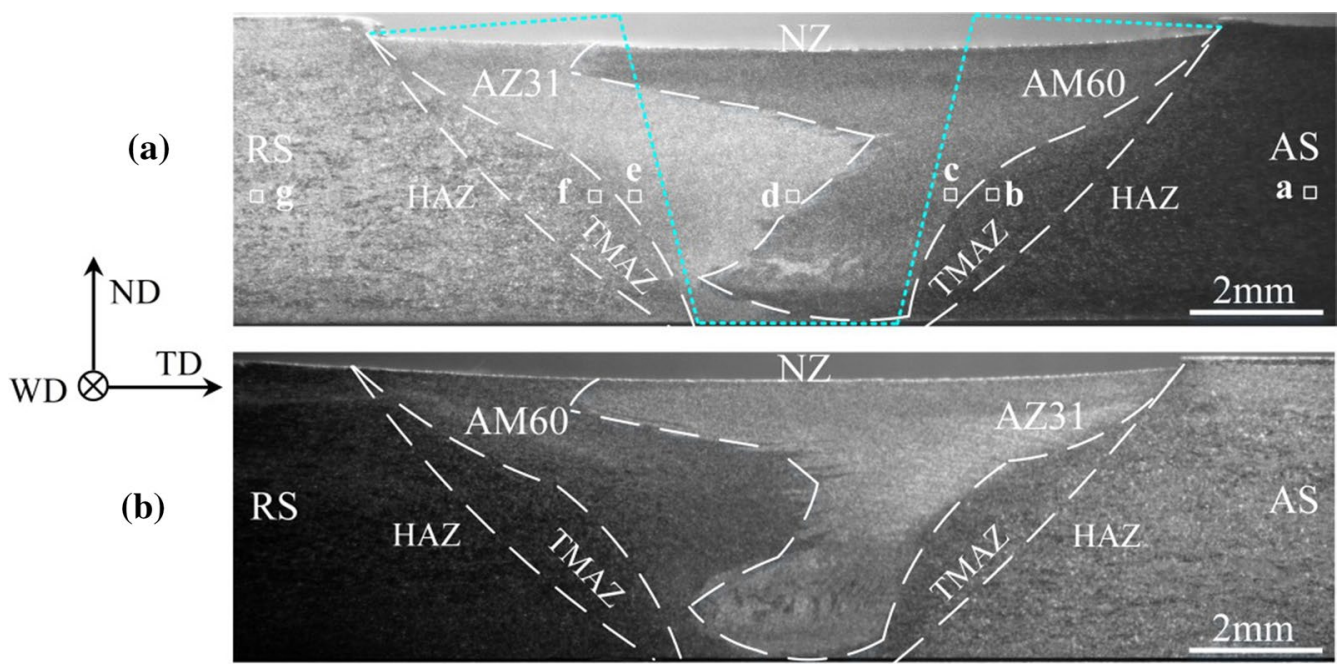

Fig. 2 Cross-sectional morphologies of the FSW joint: a AM60/AZ31 joint; b AZ31/AM60 joint

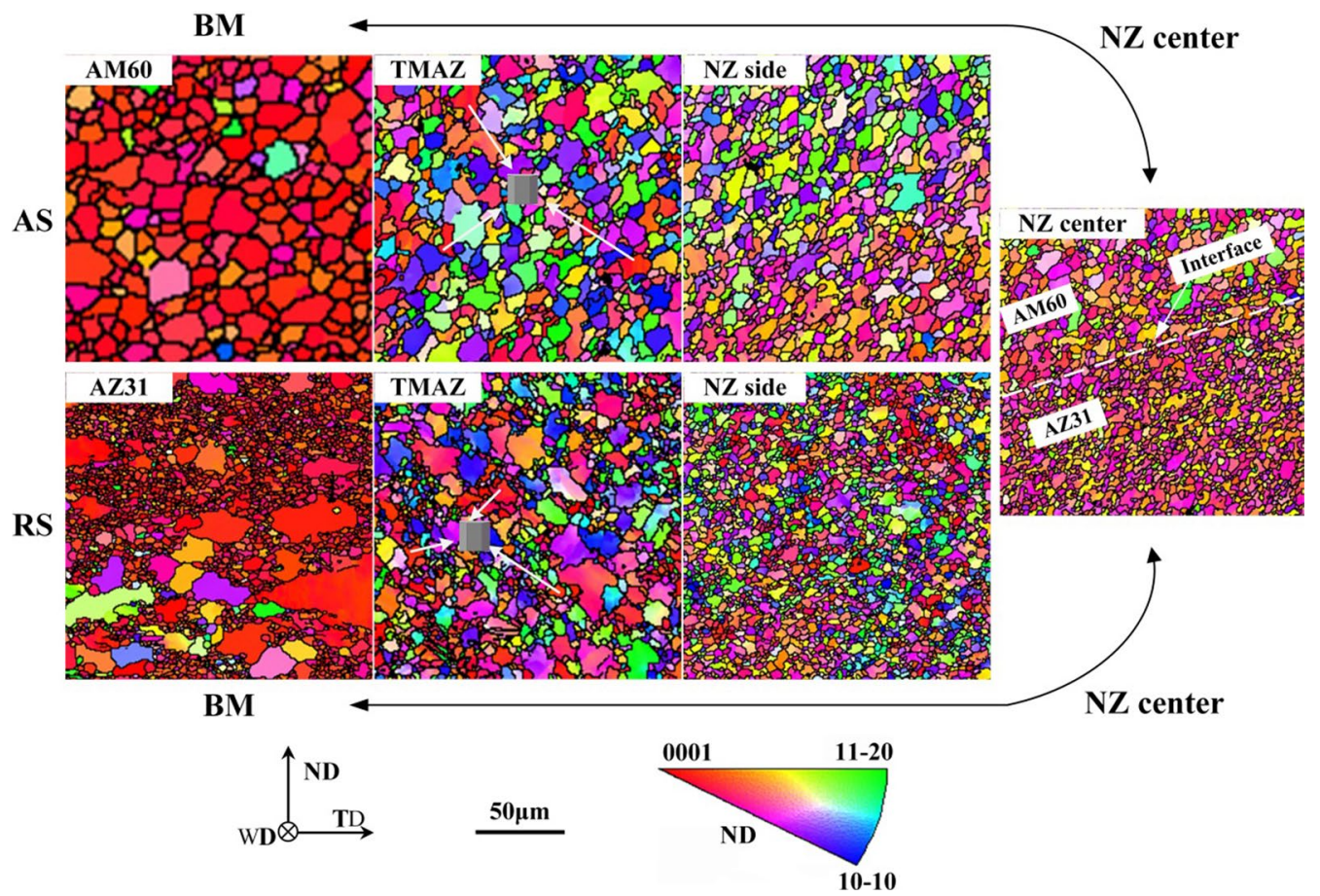

Fig. 3 Inverse pole figures of various regions of the AM60/AZ31 joint

its unique microstructure due to its obvious plastic deformation and partial dynamic recrystallization during the FSW. The metal in TMAZ was subjected to the heat generated from the stirring tool, which resulted in the metal at a high temperature state to a certain extent. Consequently, the basal slip system $[(0002)<11-20>]$, twining and even nonbasal slip systems $(\{10-10\}<11-20>$ and $\{10-11\}<11-20>)$ in TMAZ might be easily activated under the transverse and longitudinal pressures from stirring pin and the shoulder
(Fig. 4b), respectively, thereby resulting in the grain slipping and rotation $[9,18]$. These deformed grains recrystallized under the action of welding heat. However, as the transition zone of deformation, only partially deformed grains were recrystallized due to the insufficient heat in TMAZs. Furthermore, in TMAZs, the orientations of some grains approximated to the $c$-axis $\| \mathrm{ND}$, implying that they might be the un-recrystallized grains in BM (Fig. 3). In addition, the grain size of TMAZ also changed significantly. The average 


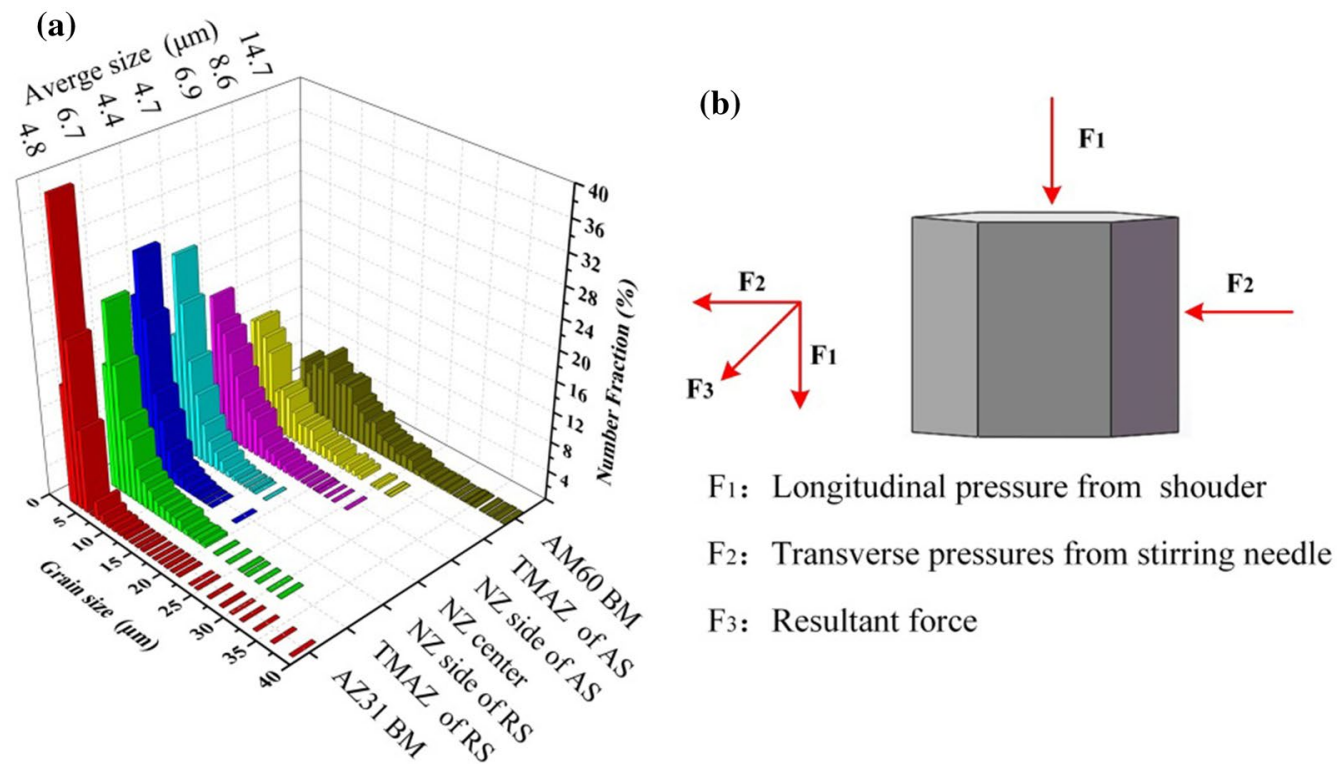

Fig. 4 Grain size distributions of the AM60/AZ31 joint a; force diagram of TMAZ during FSW b

grain size of TMAZ of AS was $\sim 8.6 \mu \mathrm{m}$ (Fig. 4a), and the grains were refined obviously compared with AM60 BM. The metal in TMAZ underwent plastic deformation under the action of stirring tool, and the internal dislocation density of the grains was increased, which was favorable for the nucleation of new grains. Meanwhile, the new grains cannot grow significantly due to the insufficient heat in this region [19], thereby causing the refinement phenomenon in it. Note that the mean grain size of TMAZ in RS increased slightly compared with that in AZ31 BM. As shown in Fig. 3, the AZ31 microstructure showed a bimodal distribution. During the deformation process of the AZ31 metal, the more dislocations were easily accumulated in larger grains, resulting in a higher probability of recrystallization nucleation. On the contrary, the smaller grains were not easy to deform and would grow to a certain extent at higher welding temperature. In the AZ31 BM, the grains with a size of less than $4.5 \mu \mathrm{m}$ accounted for $77 \%$ of the total grains. Under the actions of external force and thermal cycling, the coarse grains can be refined to a certain extent, while for the fine grains, the grain size would increase under the thermal cycling. This may be the reason for the above changes in grain size.

In Fig. 3, compared with TMAZs and BMs, there were obvious differences in grain orientation in the weld zone. The grain orientation gradually transited from $<0001>$ in the $\mathrm{BM}$ to the middle between $<0001\rangle$ and $\langle 11-20\rangle$ or $<10-10>$ in weld zone, especially in the NZ center. The microstructure in weld zone was mainly fine equiaxed recrystallized grains compared with BMs and TMAZ. During the FSW process, the grains were elongated and accumulated dislocations due to the rotating shear force of the stirring pin and then broken [20]. This provided a driving force for the recrystallization of the weld metal. Moreover, based on the previous study [20], the temperature in the FSW process can be calculated by the equation:

$T / T_{\mathrm{M}}=K \cdot\left(W^{2} /\left(V \cdot 10^{4}\right)\right)^{\alpha}$

where $T$ represents the temperature of NZ during the FSW, $T_{\mathrm{M}}$ the melting point of BMs $\left(\sim 610^{\circ} \mathrm{C}\right), V$ the welding speed $(100 \mathrm{~mm} / \mathrm{s}), W$ the rotation rate $(800 \mathrm{rpm})$. The $K$ and $\alpha$ are the constants 0.8052 and 0.0442 , respectively. In general, the recrystallization temperatures of $\mathrm{Mg}$ alloys are around $250-400{ }^{\circ} \mathrm{C}$ [21]. The calculation illustrated that the temperature obtained under this welding condition was $\sim 424{ }^{\circ} \mathrm{C}$, which can induce the recrystallization behavior of $\mathrm{Mg}$ alloys. The grains in weld zone underwent dynamic recrystallization under the actions of welding heat and intense stirring, resulting in the fine equiaxed grain. In addition, Suhuddin et al. [22] found that a large number of $\{10-12\}$ tensile twins were formed firstly in the materials in front of the stirring tool because of the extrusion of the tool. With the approaching of tool and the increasing strain, the twin boundary was gradually transformed into random high angle grain boundaries (HAGBs), while the low angle grain boundaries (LAGBs) also continuously formed in the grain, resulting in the refinement of microstructure.

By comparing the grain size of various regions (along transverse direction (TD)) in NZ, it was found that the grain size of AS was larger than that of RS, as shown in Fig. 4. Hwang et al. [23] demonstrated that the AS possessed a slightly higher temperature compared with RS during FSW. This may be the reason for the larger grain size of AS. Thus, 
the other type of material location, with AM60 in RS, was used for dissimilar FSW, as shown in Fig. 5. Notice that, whether AZ31 or AM60 on RS, the AM60 side possessed larger grain size compared with AZ31 side. This demonstrated that other factors caused the difference of grain size on both sides. Watanabe et al. [24] proposed a model to calculate the grain size of NZ. Watanabe model:

$D_{\mathrm{NZ}} / D_{\mathrm{BM}}=10^{3} \cdot Z^{-\frac{1}{3}}$

where $Z$ is a variable related to the $\mathrm{NZ}$ temperature and strain rate, $D_{\mathrm{NZ}}$ the grain size of NZ, $D_{B M}$ the grain size of $\mathrm{BM}$. It can be seen from the equation that the grain size was inversely correlated with $Z$. The $Z$ can be calculated by the equation [20]:

$Z=\dot{\varepsilon} \cdot \exp (Q /(R \cdot T))$

where $Q$ is the activation energy of lattice diffusion, $R$ represents the gas constant, $T$ is the temperature in NZ. In addition, $\dot{\varepsilon}$, during the FSW, can be calculated by the following equation [20]:

$\dot{\varepsilon}=W / 2 \cdot\left(2 \pi r_{\mathrm{e}} / L_{\mathrm{e}}\right)$

where $W$ is the rotation rate, and $r_{\mathrm{e}}$ and $L_{\mathrm{e}}$ are the effective radius and effective depth of the dynamic recrystallization region, respectively. For the same joint, $W, T, r_{\mathrm{e}}$ and $L_{\mathrm{e}}$ were fixed values; hence, $Z$ can be approximated as a fixed value. Therefore, only from the equation, the grain size of the initial microstructure had a great influence on the grain size of weld zone. Liu et al. [14] also found that the grain size of AS (ZK60) was smaller than that of RS (AZ31) in the ZK60/ AZ31 dissimilar Mg alloys FSW study. In summary, the difference of the initial microstructure and chemical composition of BMs in the dissimilar Mg alloys FSW joint was one of the important reasons for the difference of grain size of two metals in joint.
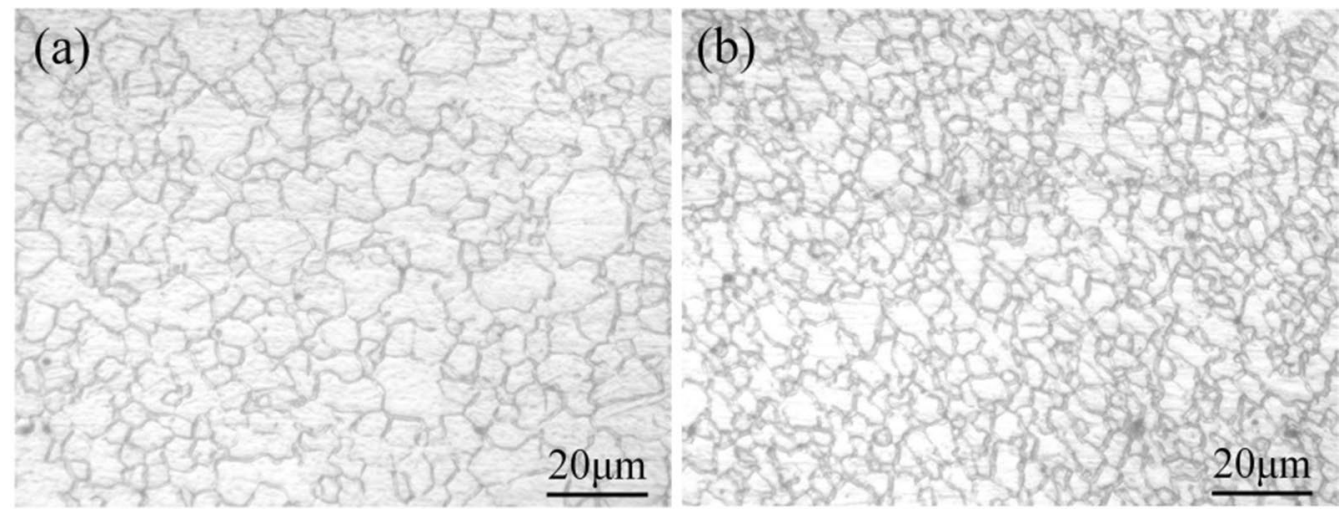

\subsection{Analysis of Grain Boundary Characterization and Recrystallization Behavior}

The distribution of grain boundaries can reflect the degree of recrystallization of various micro-regions in joint $[1,4]$. The degree of recrystallization affects the grain size, dislocation distribution, etc. of various micro-regions. Therefore, the analysis of the grain boundary characterization of various micro-regions in joint can increase the understanding of microstructure evolution in dissimilar FSW of $\mathrm{Mg}$ alloys. Figure 6 shows the grain boundary maps, the misorientation angle distributions and grain morphology maps of BMs. In the grain boundary maps, the HAGBs $\left(>10^{\circ}\right)$ and the LAGBs $\left(2^{\circ}-10^{\circ}\right)$ were represented by black and red lines, respectively. A small number of LAGBs were observed in the as-received AM60, and the percentage was only $4.7 \%$, while the percentage of recrystallized grains was up to $97.4 \%$. However, compared with AM60, the asreceived AZ31 had lower percentages of HAGBs (76.5\%) and recrystallized grains (60.9\%). This demonstrated that the as-received AM60 sheet underwent more sufficient recrystallization than the as-received AZ31 during the hot extrusion process.

The grain boundary maps, misorientation angle distributions and grain morphology maps of TMAZs are exhibited in Fig. 7. After FSW, the number of LAGBs in TMAZ increased greatly compared with that of BMs. The percentages of LAGBs in TMAZs of AS and RS increased to $18.2 \%$ and $39.5 \%$, respectively. Moreover, the number of LAGBs, especially for the grain boundaries in TMAZ with the angle $2^{\circ}-5^{\circ}$, increased obviously. During the FSW process, the TMAZ was subjected to the extrusion forces from stirring pin and shoulder; the metal in TMAZ underwent severe deformation under the external force, causing a large increase in the number of LAGBs. Concurrently, the TMAZ also absorbed the heat generated by stirring tool, and the grain would recover, recrystallize and grow under

Fig. 5 AZ31 metal a; AM60 metal b of the AZ31/AM60 joint 
(a)

Grain boundary maps
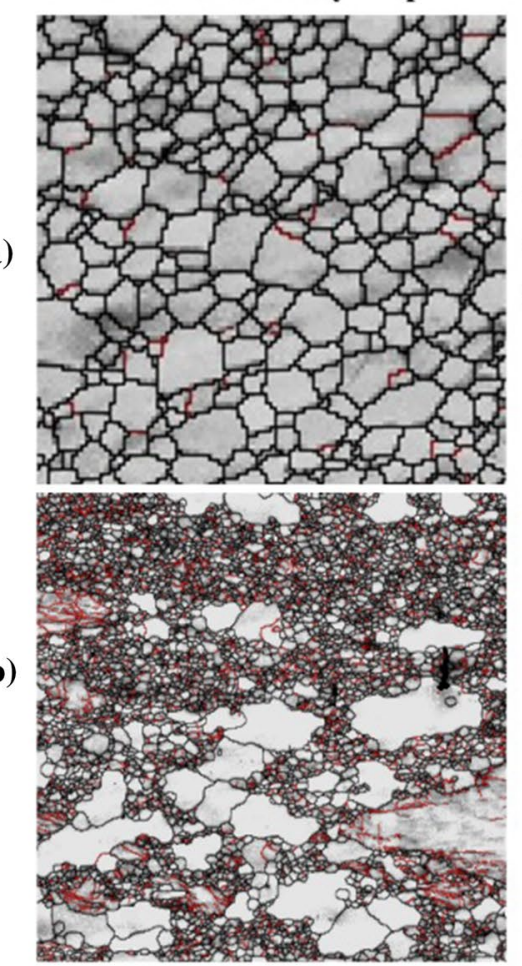

${ }_{\mathrm{WD}} \stackrel{\mathrm{ND}}{\mathrm{TD}} \stackrel{\mathbf{5 0 \mu \mathrm { m }}}{\longrightarrow}$
Misorientation angle distributions
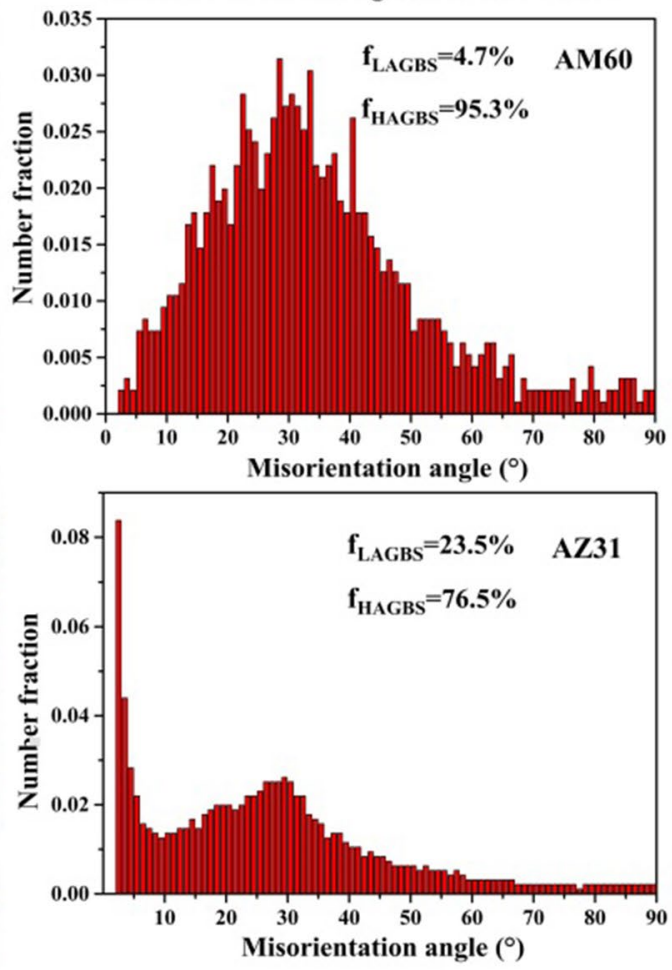

Grain morphology maps
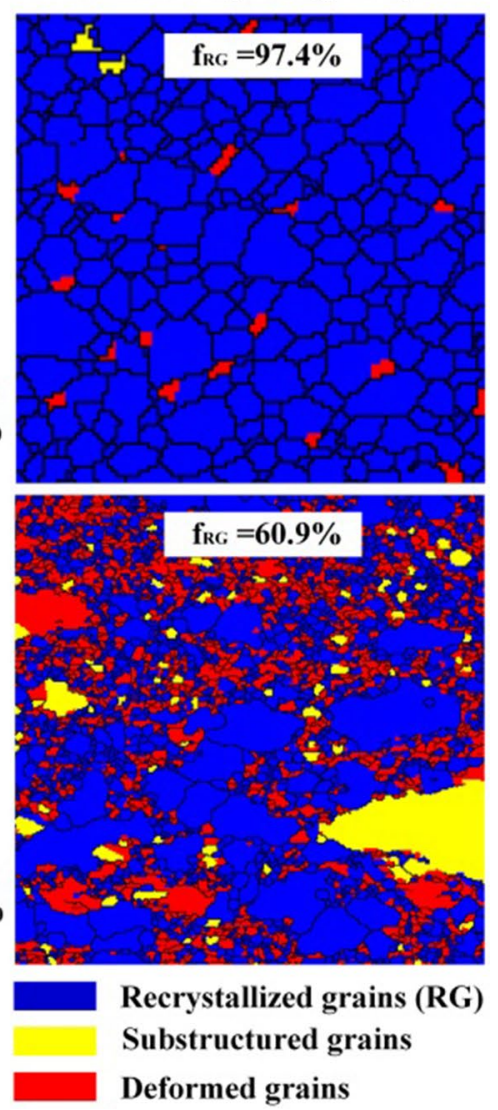

Fig. 6 Grain boundary maps, misorientation angle distributions and grain morphology maps of BMs: a AM60; b AZ31

the action of crystallization driving force. However, due to the insufficient heat in TMAZ, it cannot satisfy the complete recovery of most deformed grains, even recrystallization and growth, so a large number of LAGBs were retained in TMAZ. By comparing Fig. 7 a with Fig. 7b, it can be found that the percentages of HAGBs and recrystallized grains in the AS presented a significant increase compared with the RS. Theoretically, the higher the temperature is, the more complete recovery and recrystallization are. Hwang et al. [23] confirmed that the temperature of AS was higher than that of RS during the FSW. This may be the reason for the lower percentage of LAGBs and the higher percentage of recrystallized grains in AS. In addition, about $23.5 \%$ of the LAGBs were still retained in the initial AZ31 matrix (Fig. 6b). The existence of this part of the initial sub-grain boundaries was also the reason why the AZ31 side possessed a higher percentage of LAGBs.

Figure 8 shows the grain boundary maps, misorientation angle distributions and grain morphology maps of NZ. Compared with the AM60 BM, the various regions of weld presented a higher percentage of LAGBs and a lower percentage of recrystallized grains. Moreover, the percentage of LAGBs in both the NZ side of AS and the NZ center was lower than that in the NZ side of RS. The percentage of LAGBs was $21.7 \%, 20.1 \%$ and $16.4 \%$ for the NZ side of RS, NZ center and NZ side of AS, respectively. In contrast, the percentage of recrystallized grains increased in the order: NZ side of RS $(59.6 \%)<\mathrm{NZ}$ center $(68.4 \%)<\mathrm{NZ}$ side of AS (71.7\%). During the FSW process, the grain was elongated and accumulated dislocations under the rotating shear force of the stirring pin, and the LAGBs were continuously generated in the grain interior. Theoretically, the $\mathrm{Mg}$ alloys have low stacking fault energy [8], and the deformed grains would undergo the continuously and discontinuously dynamic and static recovery or recrystallization under the thermal cycling, resulting in the gradual absorption of dislocations and the transformation of LAGBs to HAGBs [20, 25], as shown in Fig. 9a. In addition, the $\{10-12\}$ extension twins were also produced under the extrusion force of the tool in the early stage of FSW, and those twins were eventually transformed into random HAGBs under the action of rotational shear stress and thermal cycling [20]. However, after the stirring tool moved away from the welded area, some of the LAGBs cannot absorb enough dislocations to transform into HAGBs and eventually remain in the microstructure because the FSW failed to provide a sustained 
(a)

(b)
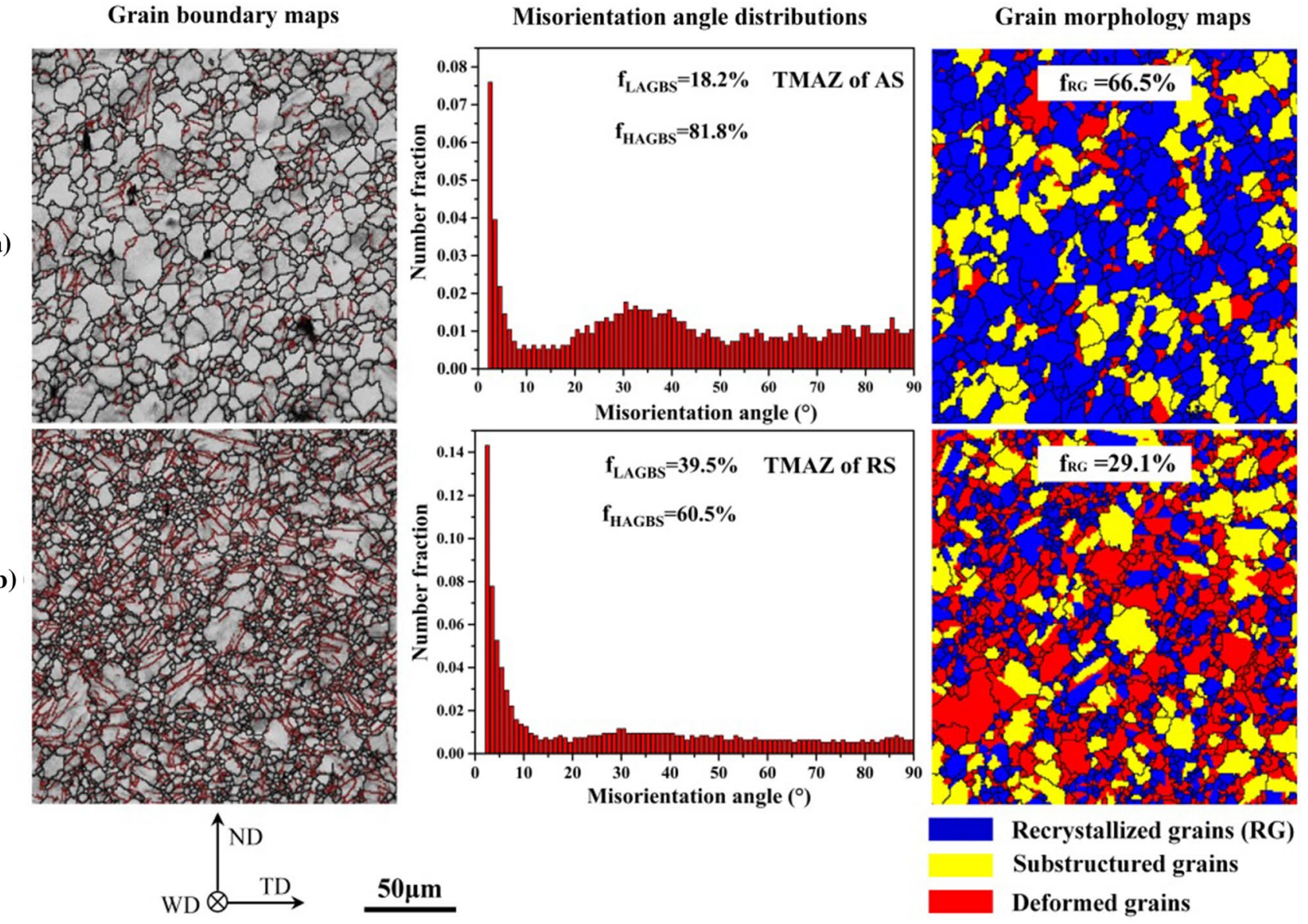

Fig. 7 Grain boundary maps, misorientation angle distributions and grain morphology maps of TMAZs: a AS; b RS

high-temperature environment. This resulted in a higher percentage of LAGBs and a lower percentage of recrystallized grains in weld. Tang et al. [26] considered that the temperature distribution of $\mathrm{NZ}$ was relatively uniform compared with other regions. However, the complex thermomechanical effect of tool movement in this region on the material would still lead to some differences in the welding temperatures of different micro-regions. The temperature of the AS was higher than that of the RS because the AS was the main force point of the stirring tool, and the closer to the edge of the NZ in the AS, the higher the temperature. Thus, the difference in temperature was one of the main reasons for the different percentages of LAGBs and recrystallized grains in each micro-region along the TD.

\subsection{Texture Evolution}

Figure 10 exhibits the texture distributions of the FSW joint. The as-received AZ31 and AM60 sheets all displayed a strong $\{0001\}$ basal texture with the grain $c$-axis vertical to the WD-TD plane, and the texture intensity was about 11.7 and 12.5 times random, respectively. This was a typical feature for extruded Mg alloy sheets. After FSW, it can be found that TMAZ exhibited lower texture intensity than BMs. The texture in TMAZ was more random, especially for the TMAZ of RS. The texture intensity of TMAZ in AS and RS was 6.0 times and 4.9 times random, respectively. The TMAZ was mainly subjected to the longitudinal and lateral pressures from the shoulder and the string pin, respectively, during the FSW. The grain deformed under the action of external force, causing the grain to deviate from the initial orientation (incline from ND to TD). Shang et al. [18] found that TMAZ also produced a large number of extension twins under external force in the FSW study of AZ31 Mg alloy. The formation of twins can induce recrystallization and change the orientation components. Meanwhile, in the region adjacent to NZ, the orientation of grain showed a continuous gradient change with the decrease of distance from $\mathrm{NZ}$ and gradually approached the edge orientation of NZ [18]. In addition, in Fig. 3, some un-recrystallized grains in BM still existed in the TMAZ. These may be the reasons for the decrease of texture intensity of TMAZ.

The change of texture in weld zone was significantly different from that in TMAZ. The texture intensity of each 
(a)

(b)

(c)
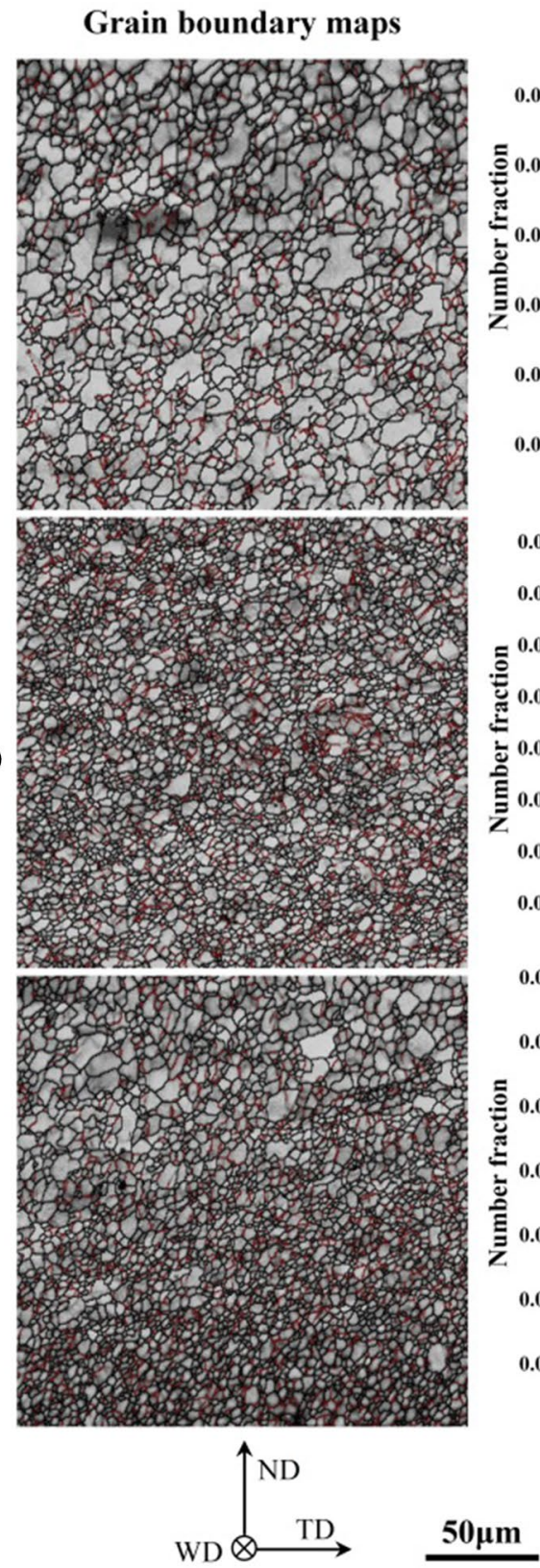

$50 \mu \mathrm{m}$
Misorientation angle distributions
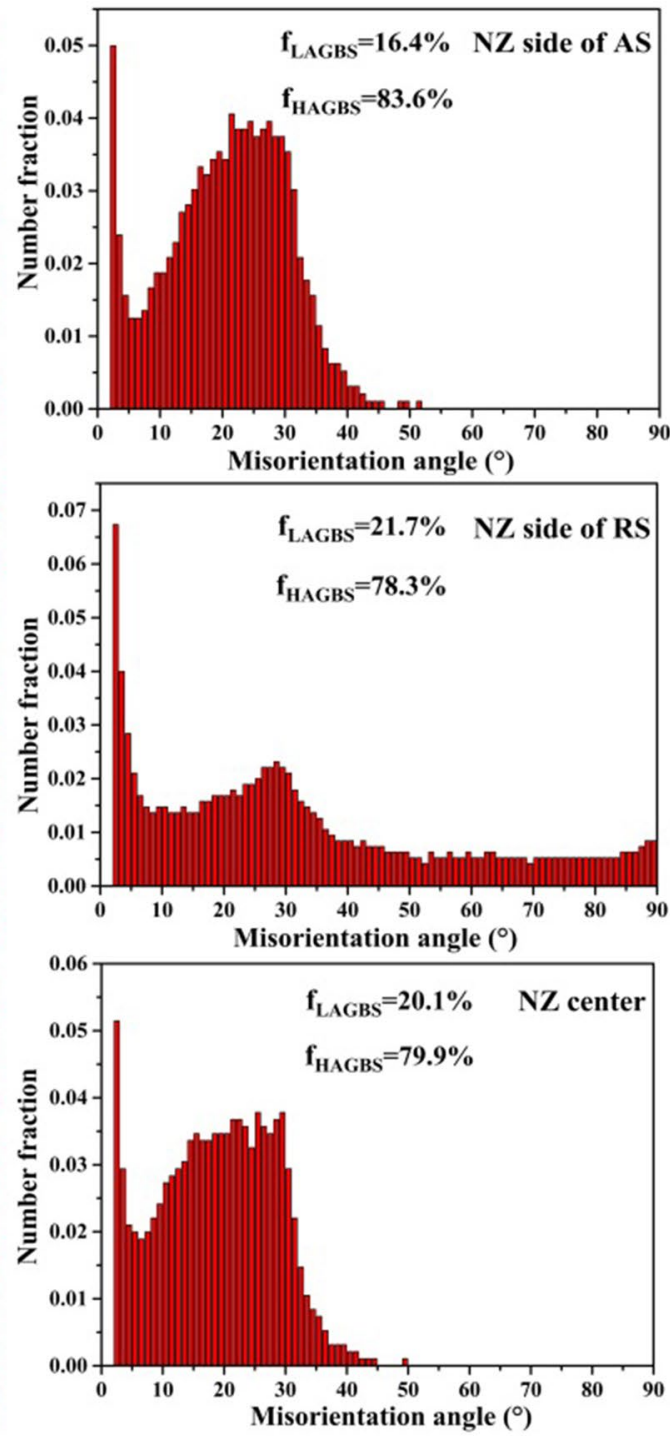

Grain morphology maps
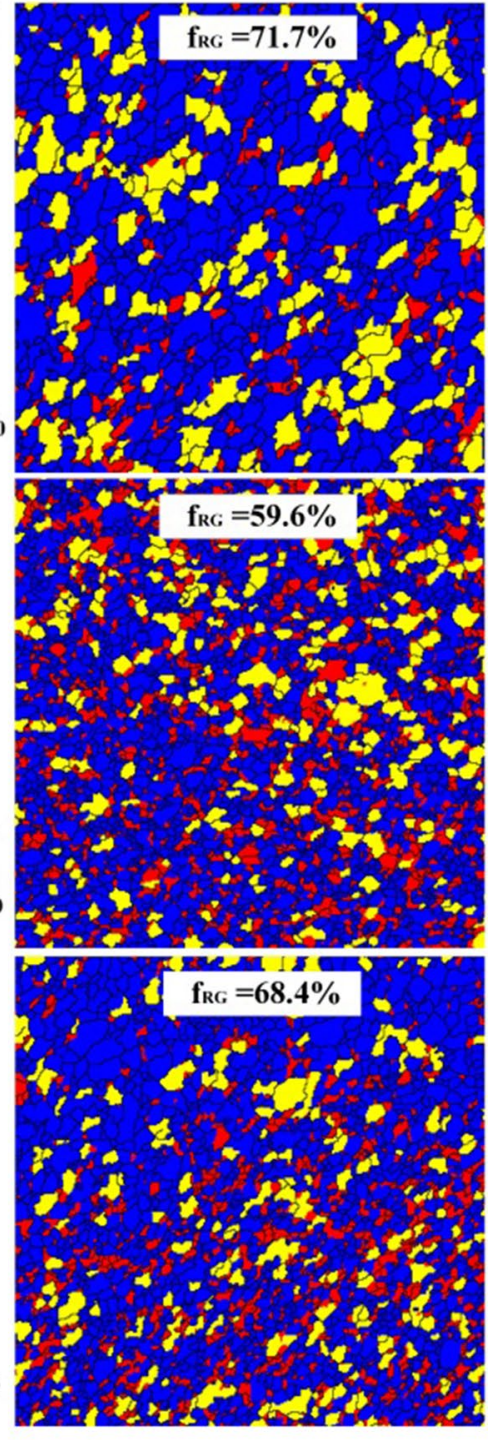

Recrystallized grains (RG) Substructured grains

Deformed grains

Fig. 8 Grain boundary maps, misorientation angle distributions and grain morphology maps of NZ: a NZ side of AS; b NZ side of RS; $\mathbf{c}$ NZ center

micro-area in weld showed an increase compared with $\mathrm{BMs}$, especially for NZ center with the texture intensity about 53.5 times random. The $c$-axis of grains in $\mathrm{NZ}$ was tilted away from ND to WD by $44^{\circ}$. The angle between the $\mathrm{TD}$ and $c$-axis in NZ center, NZ sides of RS and AS was $89^{\circ}, 50^{\circ}$ and $48^{\circ}$, respectively. The metal in NZ underwent sufficient recrystallization under the action of severe plastic deformation and thermal cycling and rotated along the cylindrical surface of the stirring pin under the rotating shear force. This enhanced the uniformity of grain orientation, resulting in an increase in texture intensity. Liu et al. [14] reported the $c$-axis of NZ was tilted away from ND to WD due to the compression stress from the shoulder. As seen in Fig. 10, the texture distribution of micro-zones in NZ was almost a straight line along TD, and there was a certain degree of symmetry between the AS and the RS. The formation of the texture characteristics in NZ was related to the dominant role of basal slip during FSW and the stacking shear layer structure produced by material laminar flow [20], as shown in Fig. 9b. 

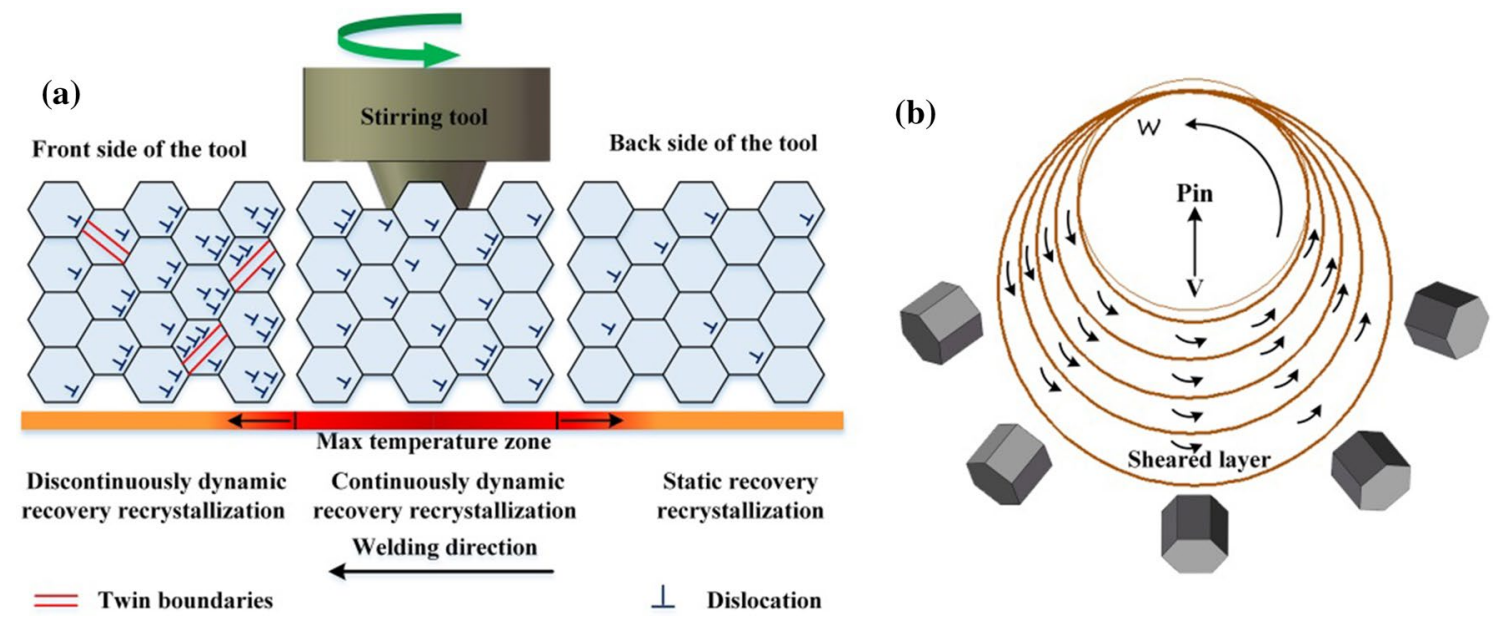

Fig. 9 Schematics of the welding process: a schematic illustration of the FSW process in NZ; b schematic illustration of the shear layer formation induced by motion of the FSW tool

AZ31 BM

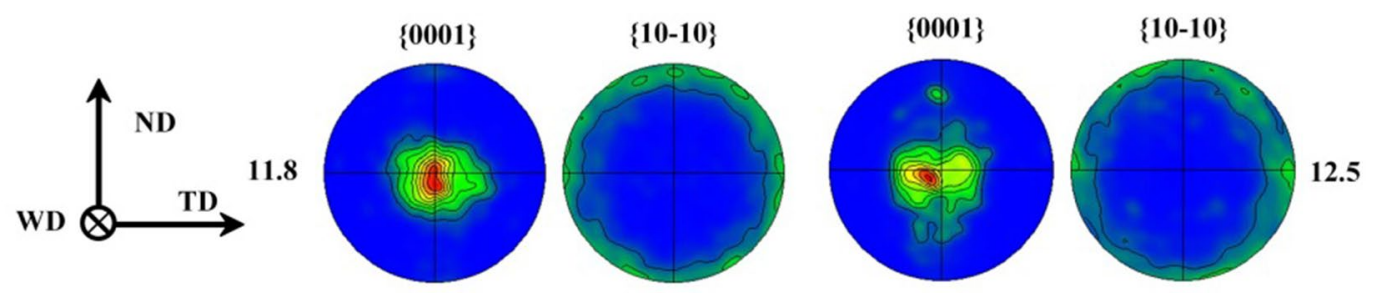

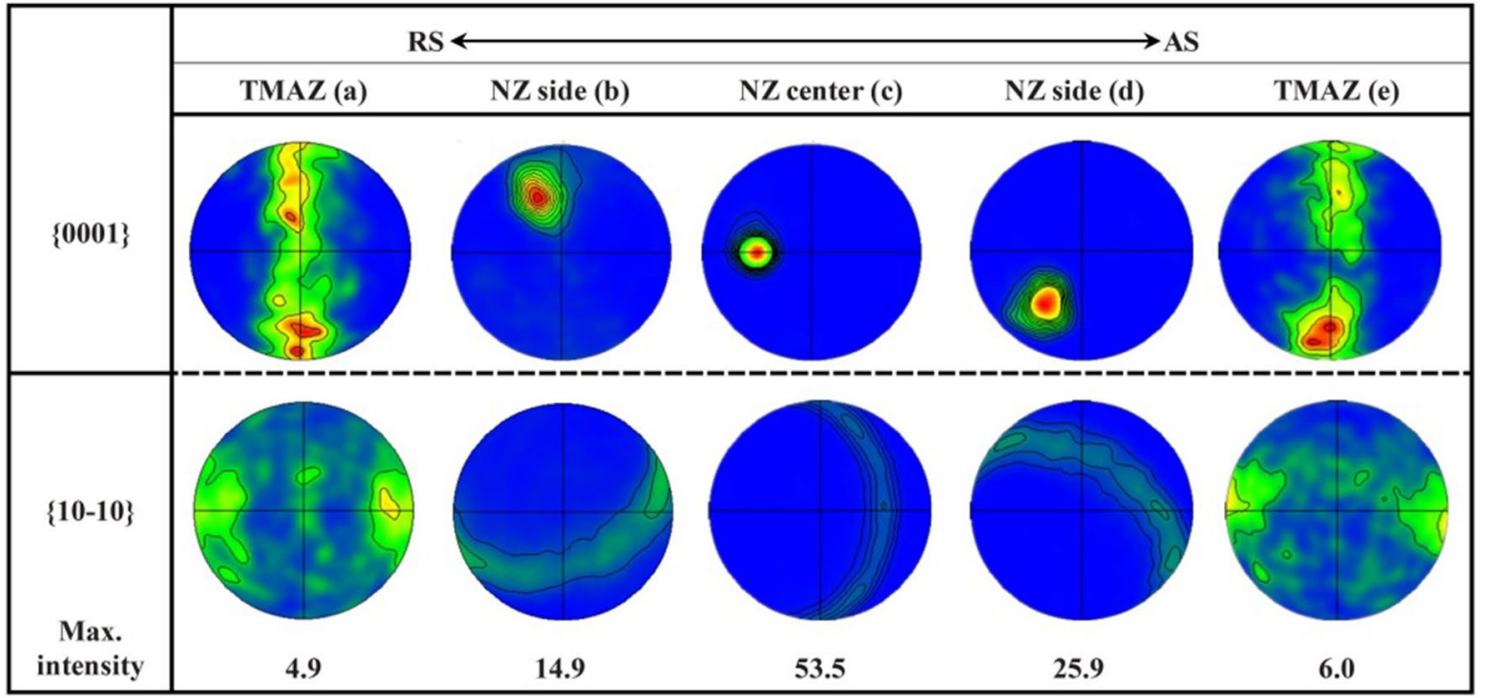

Fig. 10 Texture distributions in various regions of the AM60/AZ31 joint

\subsection{Anisotropic Tensile Properties}

Figure 11 exhibits the tensile stress-strain curves and fracture morphologies of the AM60/AZ31 joint under the three loading directions. Both AM60 and AZ31 BMs presented better tensile properties compared with the joint. This mechanical phenomenon was consistent with many earlier reports [4, 10, 13-15]. However, note that the mechanical properties (Table 1) and deformation behaviors (Fig. 11b) in the three tensile directions presented obvious differences. The elongation (EI) and ultimate tensile strength (UTS) of the FSW joint displayed an upward trend from the TD to 

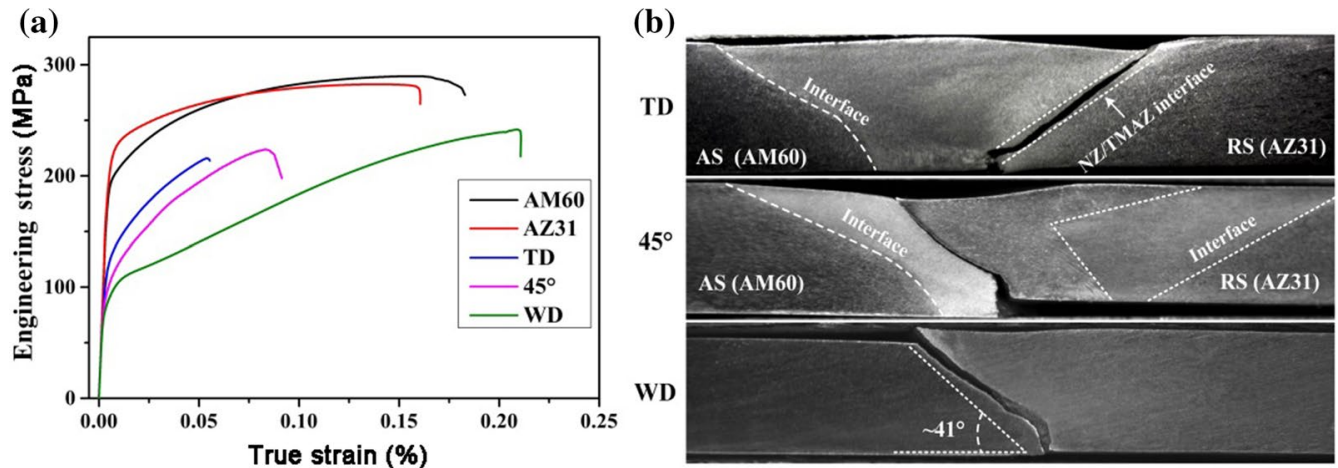

Fig. 11 a Stress-strain curves ; b fracture morphology of the AM60/AZ31 joints

Table 1 Tensile properties of the joint in the three directions

\begin{tabular}{lllr}
\hline Samples & YS (MPa) & UTS (MPa) & \multicolumn{1}{c}{ EI $(\%)$} \\
\hline TD & $116 \pm 2.9$ & $216 \pm 1.5$ & $5.9 \pm 0.6$ \\
$45^{\circ}$ & $109 \pm 2.6$ & $227 \pm 3.1$ & $9.2 \pm 0.4$ \\
WD & $100 \pm 1.8$ & $242 \pm 1.2$ & $21.2 \pm 0.5$ \\
\hline
\end{tabular}

the WD, especially for the EI. The EI and UTS increased from $5.9 \%$ and $216 \mathrm{MPa}$ in TD to $21.2 \%$ and $242 \mathrm{MPa}$ in $\mathrm{WD}$, respectively. In contrast, there was a slight decrease in yield strength (YS) from TD to WD. The YS of the three directions was $116 \mathrm{MPa}$ (TD), $109 \mathrm{MPa}\left(45^{\circ}\right)$ and $100 \mathrm{MPa}$ (WD), respectively. Moreover, the three types of tensile samples presented different fracture locations and morphologies (Fig. 11b). The fracture location of the specimen in TD was near the TMAZ/NZ interface on AZ31 side. In $45^{\circ}$ direction, however, the specimen fractured at the weld zone near the AM60 side. The specimen in WD exhibited a common oblique $45^{\circ}$ fracture morphology.

Figure 12 presents the SEM images of tensile fracture surfaces. In Fig. 12a, d, the TD sample exhibited the ductile-brittle fracture characteristic, some brittle fracture planes and small-shallow dimples were observed. However,
TD
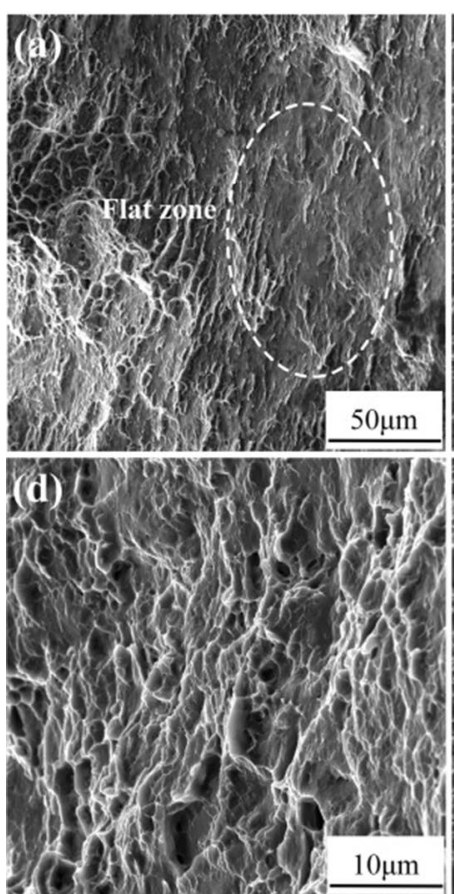

$45^{\circ}$
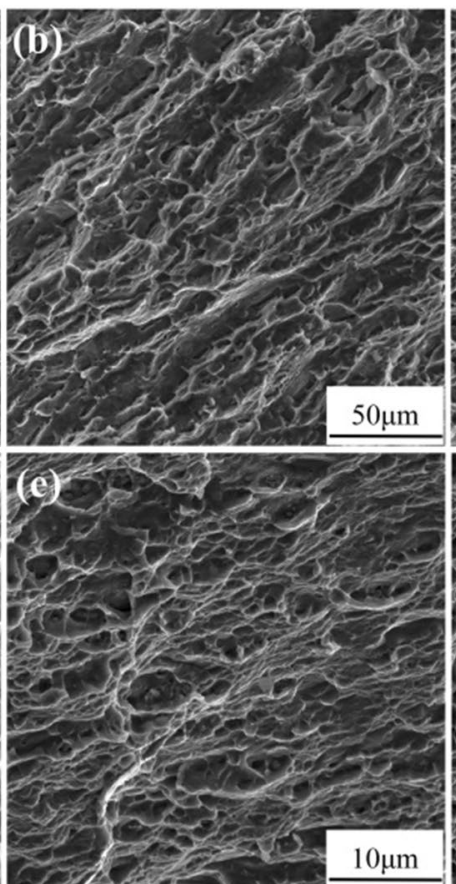

WD
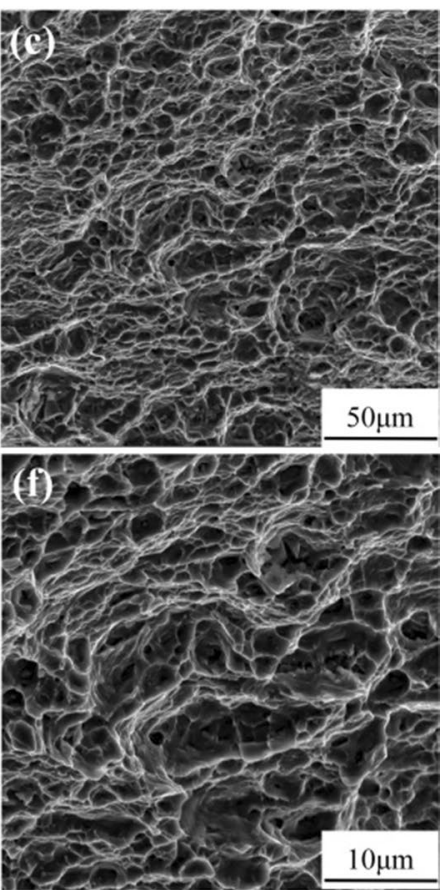

Fig. 12 Fracture morphologies of three types of tensile samples: a , d TD; b ,e $45^{\circ} ; \mathbf{c}, \mathbf{f}$ WD 
the number and depth of the dimples in the fracture surface presented a certain increase when the loading direction was $45^{\circ}$ direction (Fig. 12b, e). This also implied that the plasticity of the $45^{\circ}$ sample was better than that of the TD sample. In the WD direction, the joint surface showed the best ductile fracture morphology, and a large number of large-deep dimples were observed (Fig. 12c, f).

After FSW, the mechanical properties of the joint were lower than those of the BMs. Without loss of generality, the factors influencing the mechanical properties were primarily covered by grain size, crystalline orientation and dislocation, etc. [3]. After FSW, the grain in the weld zone was finer and more homogeneous than that in the BMs (Fig. 3), which can achieve a fine grain strengthening effect. In addition, the number of dislocations in the joint may change after FSW. The geometrically necessary dislocation density $\left(\rho^{\mathrm{D}}\right)$ of varies regions was calculated based on the EBSD data to show the changing trend of dislocation in joint, and the $\rho^{\mathrm{D}}$ can be expressed by the equation [27]:

$\rho^{\mathrm{D}}=2 \theta /(u \cdot b)$

where $u$ is the unit length and $b$ is the Burgers vector of $\mathrm{Mg}$; values $(\theta)$ above the defined threshold $\left(2^{\circ}\right)$ are eliminated. The calculation results demonstrated that the dislocation density in joint increased to some extent compared with that in BMs (Fig. 13), which can achieve a certain work hardening effect. In fact, however, the YS of the joint was still lower than that of the BM, which indicated that there were other negative factors affecting the performance of the joint. Basal slip and extension twinning have high room-temperature activation ability because of their low critical resolved shear stress [3]. The activation of these two deformation mechanisms largely depends on texture. Then, the Schmidt factors (SFs) for these two deformation mechanisms in the three loading directions of the joint are briefly displayed in Figs. 14 and 15. The average value of SF in each area of the joint was calculated to more intuitively observe the change of SF in the joint, as shown in Fig. 16. In all three directions, the highest SF for basal slip in some areas of the joint was not less than 0.4, which was significantly higher than that of BM ( 0.2). Although the grain in weld was refined and the dislocation density in joint was increased to some extent, the texture in the joint was changed significantly. That was, the hard orientation for the basal slip in BM was transformed into the soft orientation in joint. This resulted in a significant reduction in the YS of the joint. In addition, Shang et al. [28] demonstrated that the premature failure and poor tensile properties were the result of the severe strain location in joint. There were obvious fluctuation of SF values in the

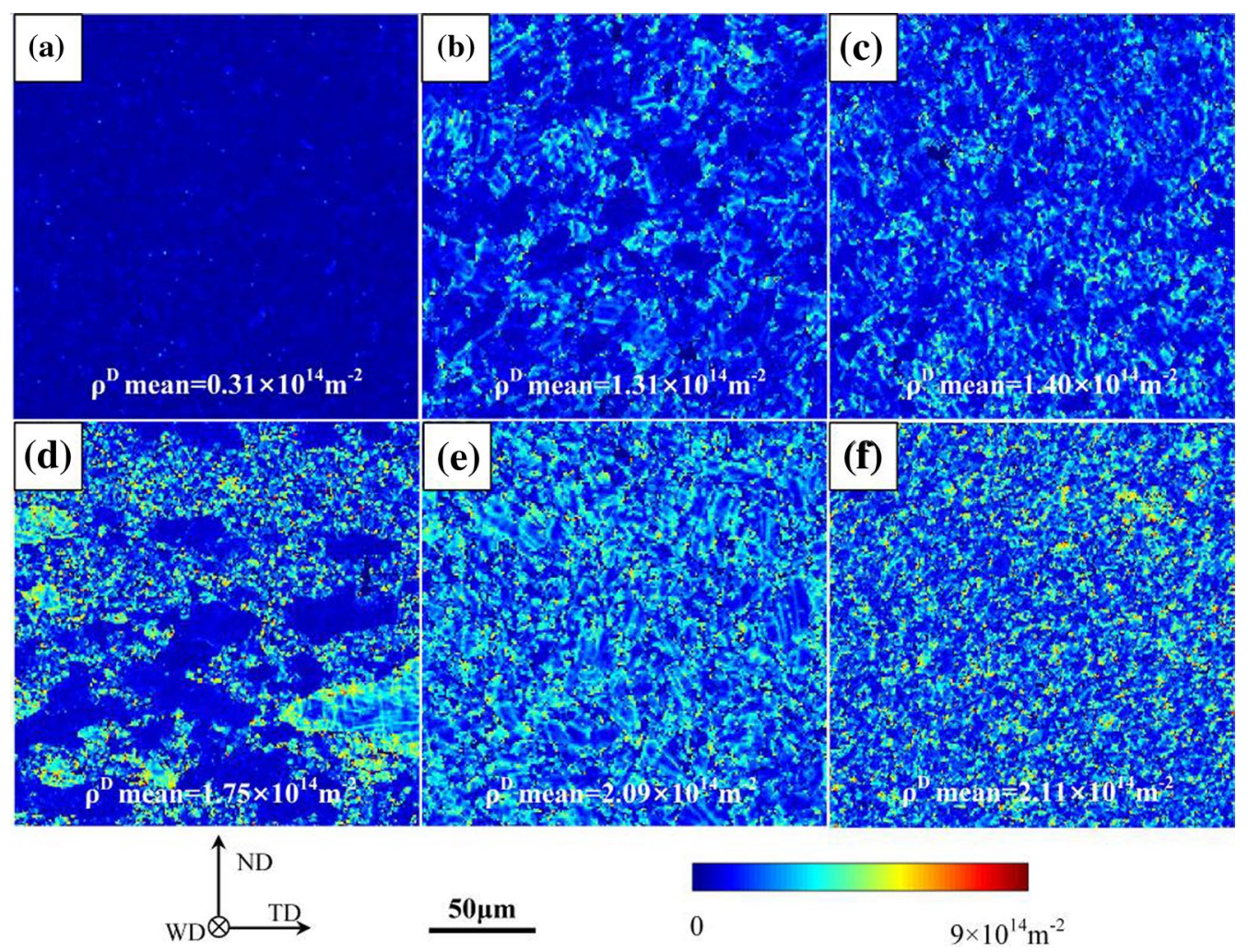

Fig. 13 Dislocation density mapping based on the EBSD data in a AM60, b TMAZ of AS, $\mathbf{c}$ NZ side of AS, $\mathbf{d}$ AZ31, e TMAZ of RS, f NZ side of RS 


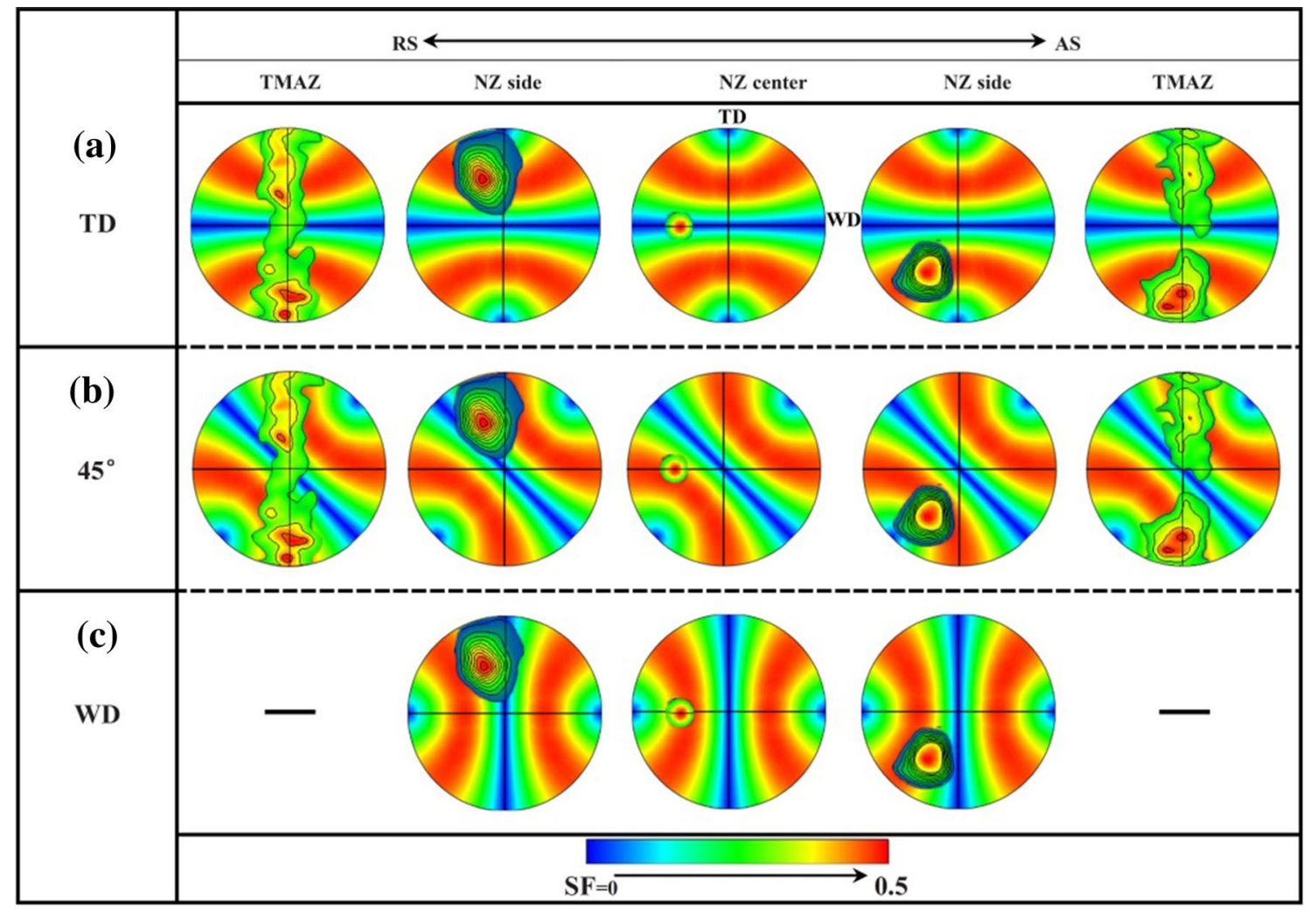

Fig. 14 SFs distributions in various regions of the joint: (0001) pole figures show the calculated SF for basal slip

tensile samples (Fig. 16), which exacerbated the joint strain localization, resulting in the lower joint EI and UTS.

Additionally, the mechanical properties of the joints in the three loading directions showed significant anisotropy. The YS of three directions was $116 \mathrm{MPa}$ (TD), $109 \mathrm{MPa}$ $\left(45^{\circ}\right)$ and $100 \mathrm{MPa}$ (WD), respectively. Many studies [29-31] demonstrated that the texture largely affected the tensile anisotropy of $\mathrm{Mg}$ alloys. Thus, for the same joint, the special textures in joint may play a key role in affecting the tensile anisotropy. Recalling Fig. 16, the maximum SF for basal slip and extension twinning in the joint showed a slight increasing trend from TD to WD. This should be the main reason why the YS of the joint presented a slight downward trend.

Compared with the YS, the UTS and EI of the joint gradually increase from TD to WD. Earlier reports $[3,18$, 28] showed that the lower UTS and premature fracture of the FSW joints were attributed to strain localization in joints. Recalling Fig. 11b, compared with the TD and $45^{\circ}$ directions, the tensile specimen showed more homogeneous deformation in the WD direction. The homogeneous deformation was conducive to the higher EI of the sample, and it was conducive to the continuous work hardening of the sample, thereby exhibiting higher UTS. Compared with the other two directions, the texture fluctuation of the weld zone along the WD direction was smaller, which reduced the strain localization. In addition, note that the EI of the WD sample was also significantly higher than that of the BMs. This result was consistent with that of previous studies [32, 33]. Liu et al. [32] obtained high-performance $\mathrm{Mg}-9 \mathrm{Li}-1 \mathrm{Zn}$ alloy by friction stir processing. EI increased from $18 \%$ of BM to $28 \%$ of weld metal. They believed that grain refinement and deflection of grain $c$-axis toward ED direction were the main factors leading to this result. Raja et al. [33] mentioned that friction stir processing can effectively improve the performance of $\mathrm{Mg}-\mathrm{Al}-\mathrm{Zn}$ alloy. EI increased from $0.8 \%$ of BM to $4.7 \%$ of weld metal. The reasons were attributed to the grain refinement and the broken second phase. After FSW, the grain size of weld zone was refined, and the microstructure was more homogeneous than the AZ31 BM. In addition, the $c$-axis of grains in weld zone was inclined from the ND (BM) to the ED by $\sim 44^{\circ}$. This led to an increase in the SFs for basal slip, which was conducive to the improvement of plasticity. As a result, these factors may result in high EI of WD samples.

Furthermore, three kinds of tensile samples after fracture were subjected to OM observation and EBSD analysis, as shown in Figs. 17 and 18. It was found that a certain number of extension twins (Fig. 17) were distributed in all the parallel sections of the WD sample. In contrast, 


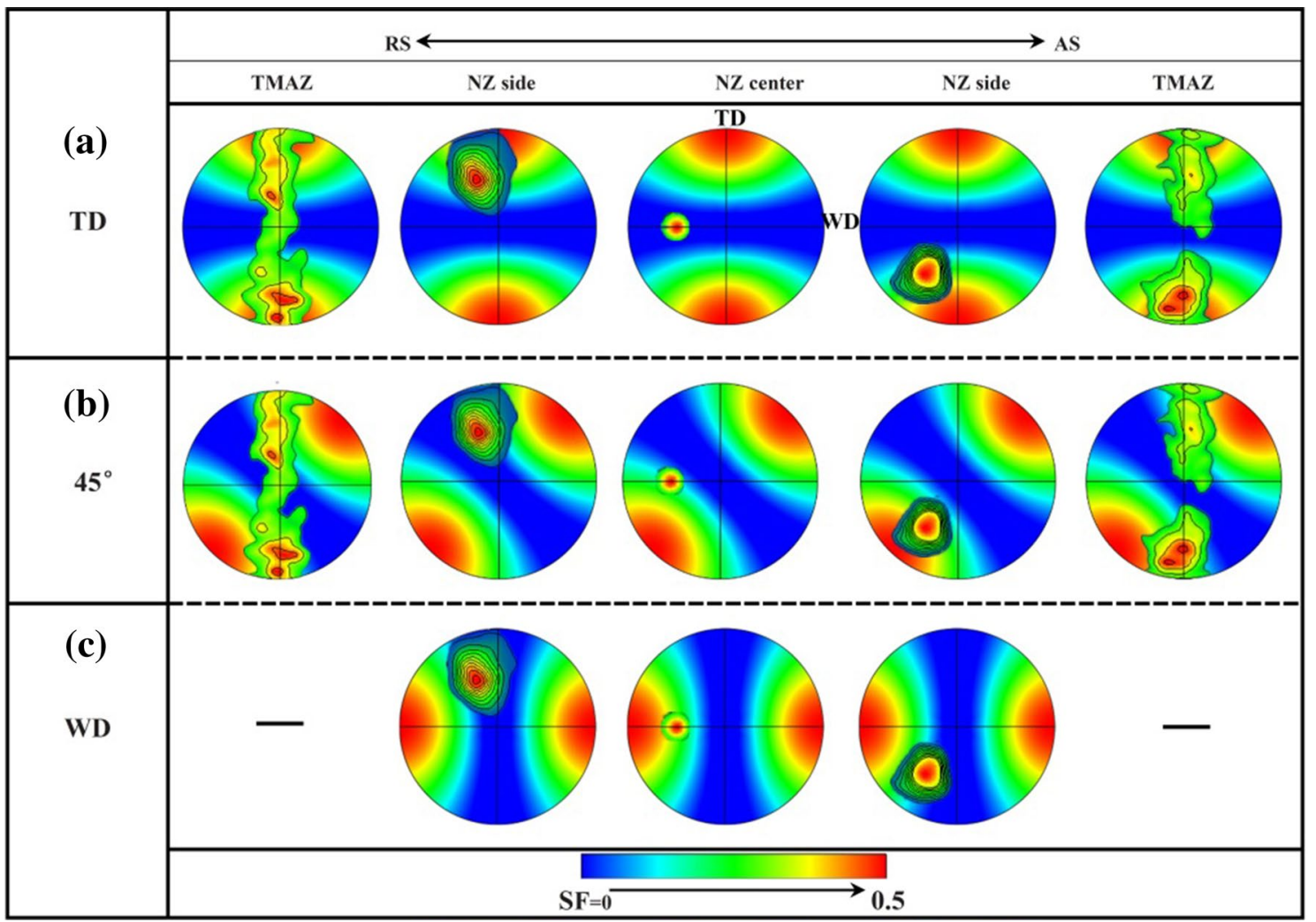

Fig. 15 SFs distributions in various regions of the joint: (0001) pole figures show the calculated SF for extension twinning

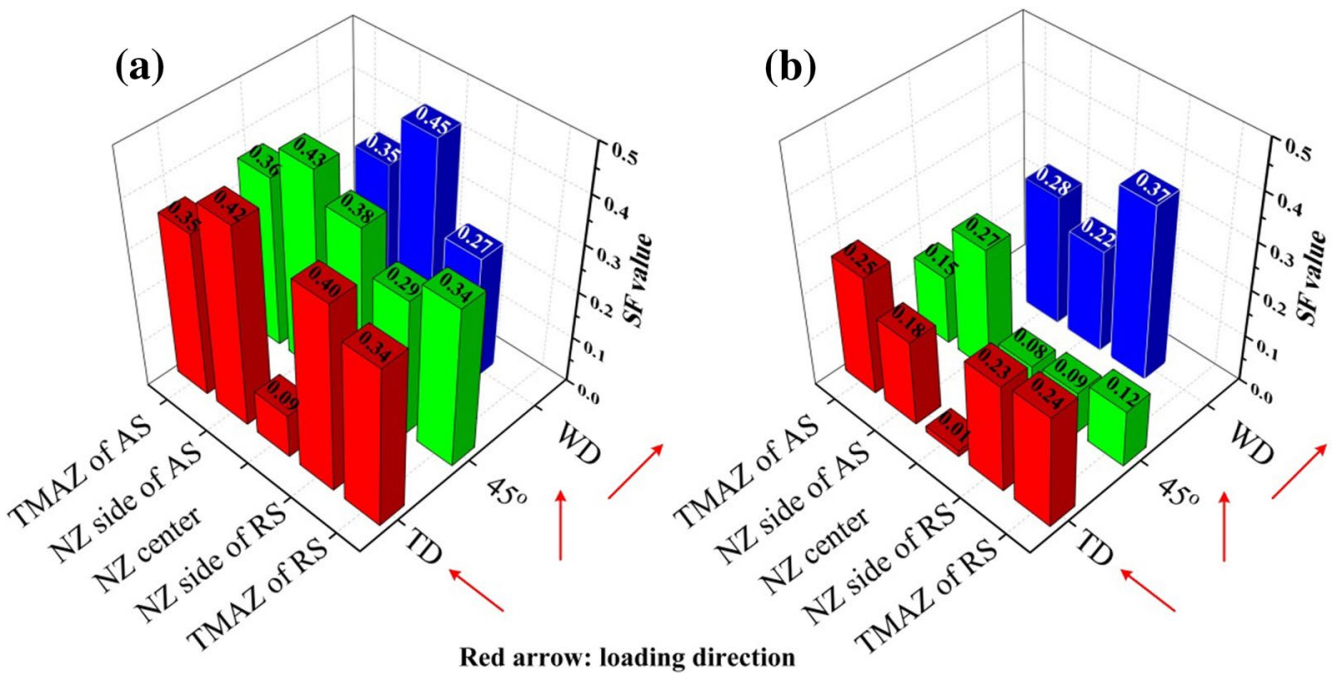

Fig. 16 Mean SF values in various regions for $\mathbf{a}$ basal slip ; b extension twinning

extension twins were observed only near the TMAZ/NZ interface for the TD sample (Fig. 18a). In the $45^{\circ}$ sample, there were a certain number of twins near the fracture edge and the NZ/TMAZ interface on the AM60 side (Fig. 18b). The formation of extension twins was accompanied by the release of stress [3]. The appearance of more extension twins in the WD sample may further promoted its higher UTS and EI. Additionally, the sample in $45^{\circ}$ direction showed higher UTS and EI compared with that in the TD direction. The inhomogeneous texture distribution was considered to be the main factor causing the deterioration of joint performance. As shown in Fig. 16, the fluctuation 


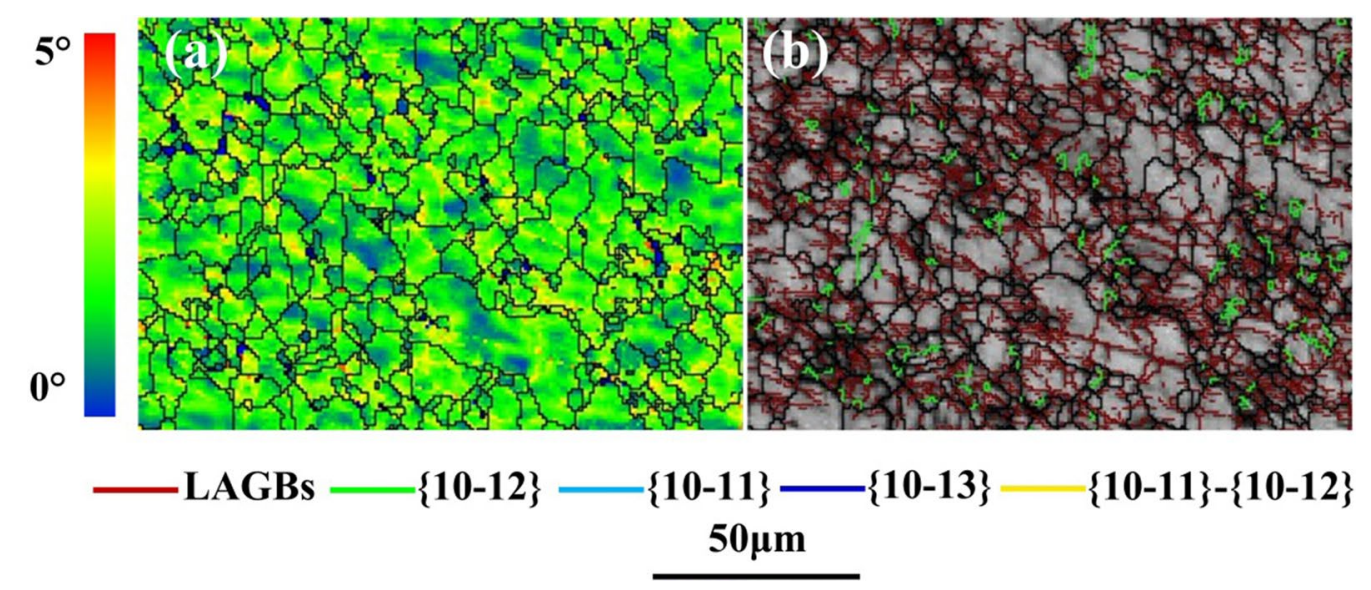

Fig. 17 EBSD maps of WD samples after fracture: a kernel average misorientation map; $\mathbf{b}$ grain boundary map

(a) TD
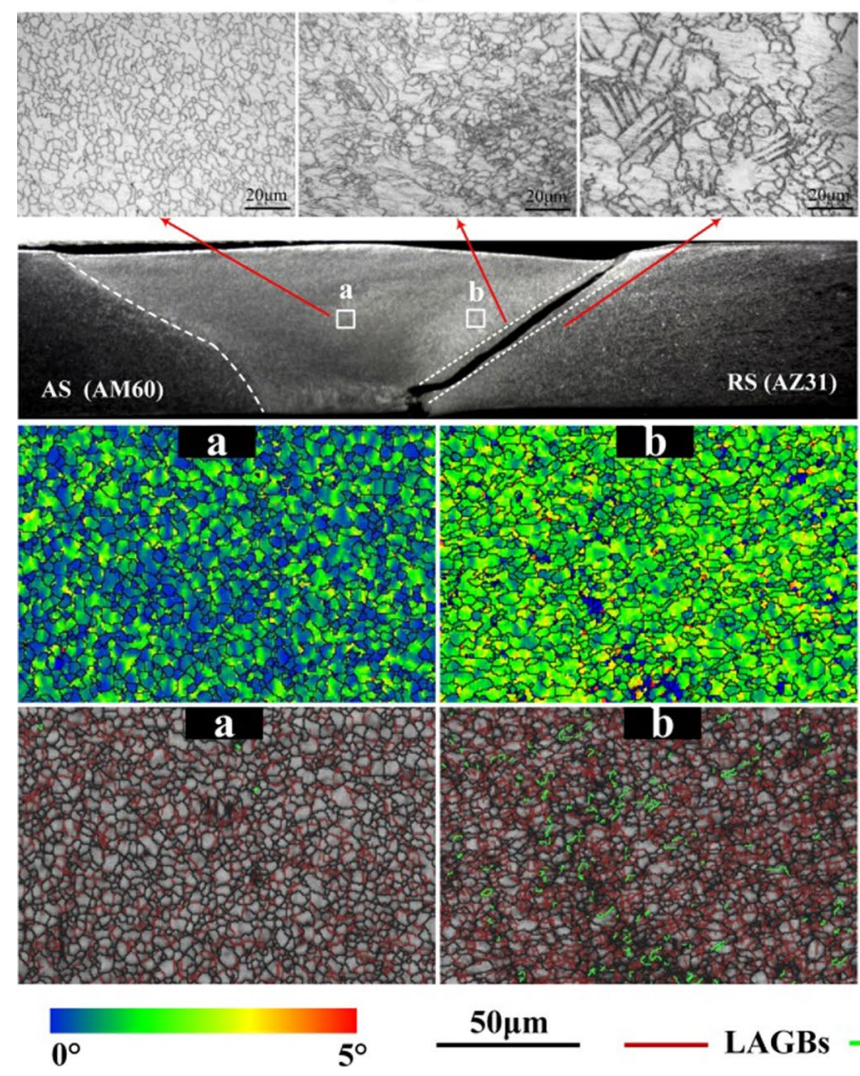

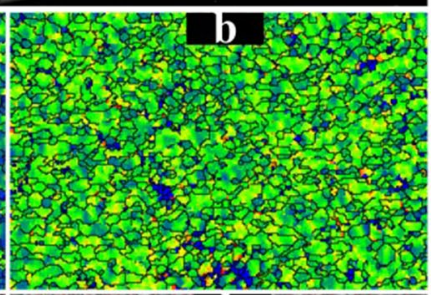

b

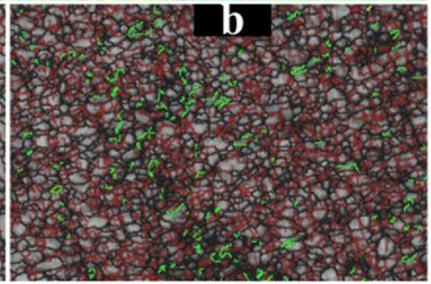

$50 \mu \mathrm{m}$
LAGBs
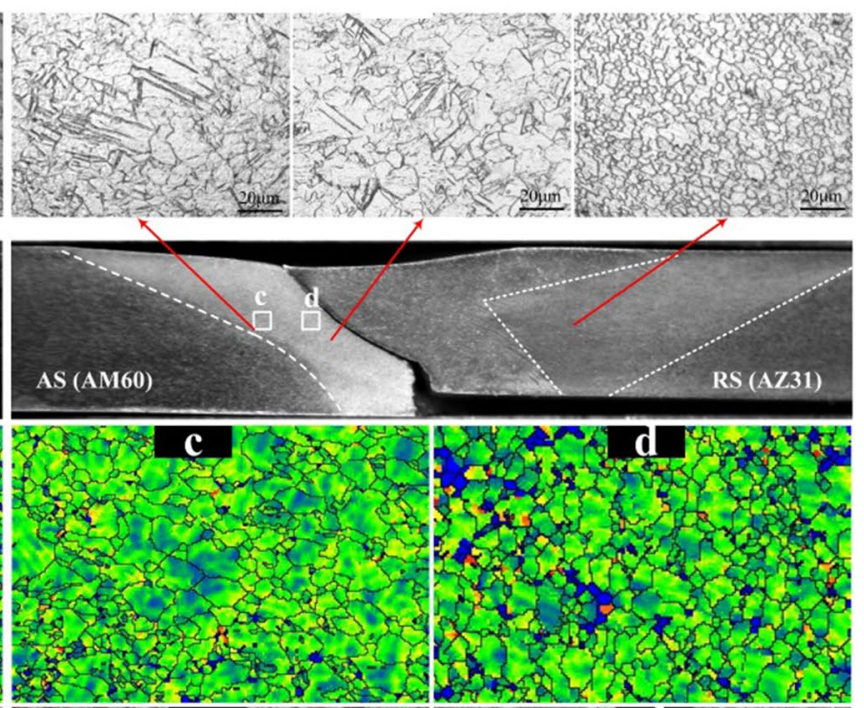

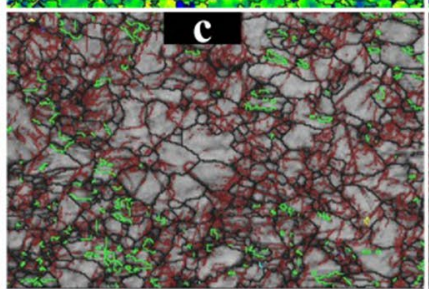

$\{10-12\}$

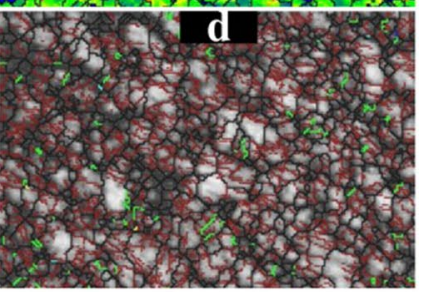

$\{10-13\}$

$\{10-11\}-\{10-12\}$

Fig. $18 \mathrm{OM}$ and $\mathrm{EBSD}$ maps of $\mathbf{a} \mathrm{TD} ; \mathbf{b} 45^{\circ}$ samples after fracture

of the SF in TD direction was larger than that in $45^{\circ}$ direction, especially for basal slip. The SF value of NZ center increased from 0.09 to 0.38 along the $45^{\circ}$ direction compared with the TD direction. During the transverse tension process, the localized strain in the joint was easily caused by the inhomogeneous distribution of SF in the joint, which would reduce the mechanical properties of the joint. When loading was exerted along the $45^{\circ}$ direction, the SF fluctuation of the joint was weakened. This result suggested that the joint strain no longer limited at the position near the TMAZ/NZ interface, so that the sample exhibited relatively higher EI and UTS. 
In addition, the second phase is also one of the factors that affect the performance of the joint $[14,16]$. The second phase particles in the two BMs and NZ were examined by SEM, as shown in Fig. 19. After FSW, the second phases in NZ were refined clearly. Theoretically, the fine second phases would contribute to the improvement of the mechanical properties of joints. However, the YS of the three kinds of samples was lower than that of the BMs. This indicated that the second phases in nonprecipitation-hardened $\mathrm{Mg}$ alloys had limited effect on the mechanical properties of the joints.

In summary, the dissimilar FSW joint showed obvious tensile anisotropy was mainly attributed to the fluctuation of the SFs, while the SFs were determined by the texture in joint. Therefore, by analyzing the texture distribution in joint, it is better to predict the plastic deformation behavior of joint in different directions, and the weak direction of joint can be selectively avoided when the workpiece is stressed.

\subsection{Fracture Mechanism Analysis}

The three types of specimens exhibited various fracture morphologies and locations. Among them, the WD sample exhibited a conventional $45^{\circ}$ fracture morphology, and no more analysis was conducted. Here, the samples in TD and $45^{\circ}$ directions were mainly compared and analyzed.

As presented in Fig. 18a, the fracture position of the TD sample was near the NZ/TMAZ interface on AZ31 side. EBSD analysis demonstrated that the strain degree and the number of LAGBs in the NZ center were significantly lower than those in the NZ/TMAZ interface. This also proved that there was a significant inhomogeneous deformation in the joint. In our previous study [3], the fracture mechanism in the TD direction was discussed mainly. The main summaries were as follows. First, the region near the NZ/TAMZ interface was the soft orientation for basal slip and extension twinning. This made the joint deformation preferentially concentrate in this region. Further, compared with the NZ/ TMAZ interface on the AM60 side, there was a higher dislocation density and strain near the AZ31 side interface. The existence of these dislocations can easily cause entanglement between dislocations and become the source of cracks. Finally, the grain size fluctuation between the TMAZ and the NZ in AZ31 side was significantly higher than that in the AM60 side, which was more likely to exacerbate the deformation incompatibility on both sides of NZ/TMAZ in the AZ31 side. The above factors led to the fracture of TD sample near the NZ/TMAZ interface on AZ31 side.

The $45^{\circ}$ specimen fractured at the weld zone near the AM60 side, as shown in Fig. 18b. First, the SF of each region in joint was analyzed. As shown in Fig. 16, the area near the TMAZ/NZ interface of AM60 side possessed high SF value for basal slip and extension twinning and large grain size, and the vicinity of this area may be a weak area of the joint. However, the actual fracture occurred in the weld zone, as shown in Fig. 18b. This indicated that other factors affected the initiation of cracks in joints. Liu et al. [34] found that the metal in lower part of the dissimilar FSW ZK60/AZ31 joint had poor tensile properties. Mironov et al. [35] mentioned that the failure was shown to be initiated at the weld root in the tensile deformation of FSW AZ31 joint. The failure of joint may be initiated from the lower part of weld. Therefore, the $45^{\circ}$ sample was subjected to a tensile test, and the loading was stopped before the fracture to determine the crack initiation position. It was found that cracks started from the bottom of weld, as shown in Fig. 20a. Further, the microstructure at the bottom of weld was observed by using OM (Fig. 20b). There was an obvious metal mixing at the bottom of weld, and the grain size of AM60 metal was larger than that of AZ31 metal. Generally, the deformation ability of large grain is different from that
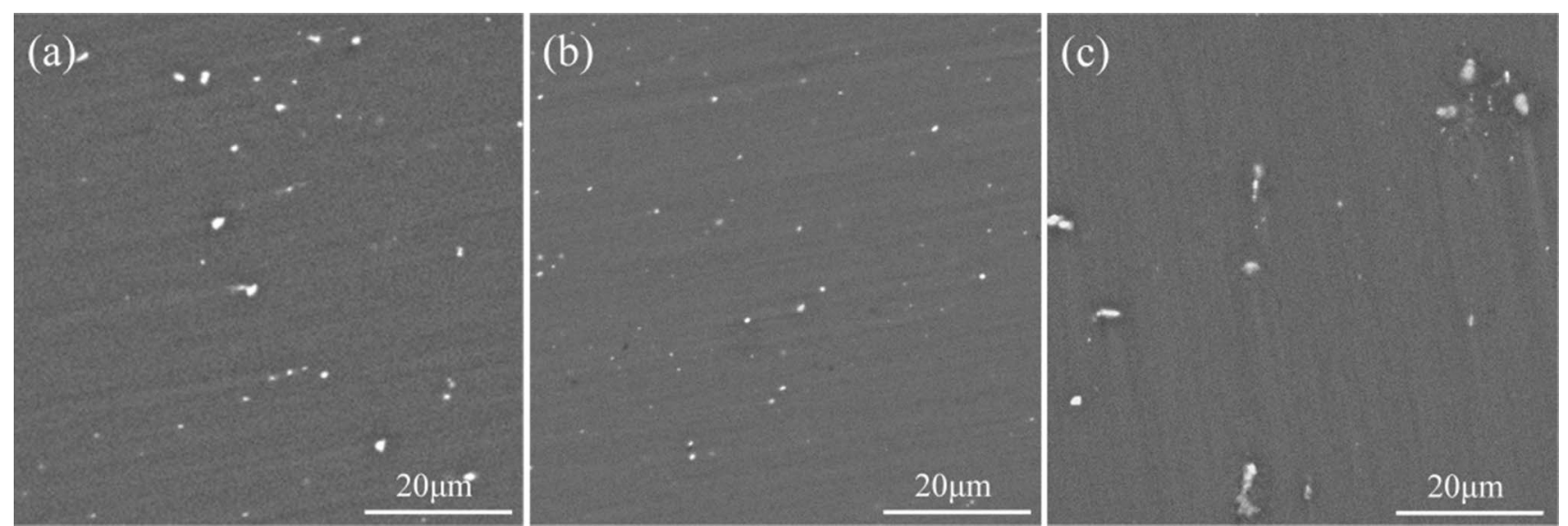

Fig. 19 SEM images showing second phases: a AZ31 BM; b NZ; c AM60 BM 


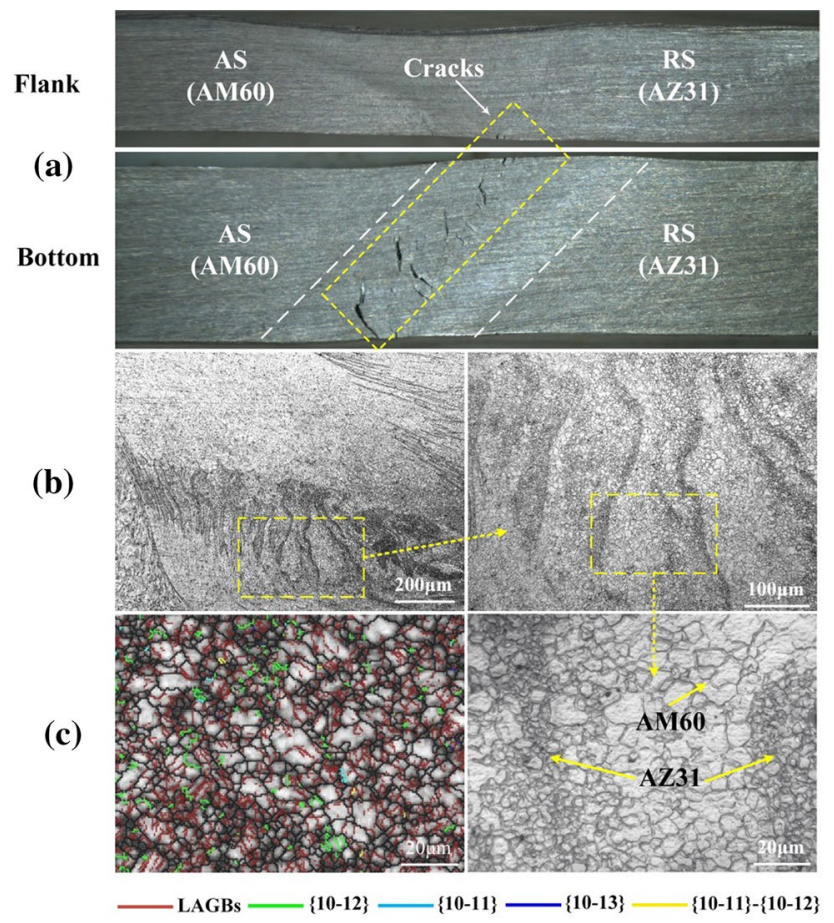

Fig. 20 a $45^{\circ}$ samples before fracture, b OM ;c EBSD maps of joint bottom

of small grain. This will aggravate the incompatibility of deformation between micro-regions during the deformation process, causing stress concentration and then becoming a source of cracks [3]. Moreover, the SFs for basal slip from the NZ center to the NZ/TMAZ interface on AM60 side were high $(\sim 0.4)$. This could cause the large metal deformation from the NZ center to the NZ/TMAZ interface, as shown in Fig. 20a. As a result, in the case of higher SF for basal slip and severe metal mixing, the metal between the $\mathrm{NZ}$ side and NZ center at the weld bottom became a source of cracks due to stress concentration. In addition, a certain number of extension twins were observed at the bottom of the sample after fracture by EBSD analysis (Fig. 20c). The possibility of extension twinning being activated was smaller due to the lower SF value for extension twinning at most locations of the weld. However, extension twins were still found near the bottom of NZ center, which indicated that there was a strong stress concentration at the bottom during the deformation. Furthermore, the crack gradually expanded from the bottom to the AM60 side. This was because the SF value on the AM60 side was higher than that on the AZ31 side as a whole; meanwhile, the AM60 side possessed larger grain size compared with the AZ31 side. This resulted in more severe deformation in AM60 side of the joint, which in turn caused the crack to propagate toward the AM60 side. The above may be the reason why the sample in $45^{\circ}$ direction displayed the above-mentioned fracture phenomenon.

\section{Conclusions}

The microstructure, texture distribution, tensile anisotropy and fracture behaviors of AM60/AZ31 dissimilar FSW Mg joint were investigated and discussed. The following conclusions can be drawn:

1 After FSW, the grain orientations in NZ fluctuated obviously, and the grain orientation from the NZ side to NZ center gradually approached from $\langle 11-20\rangle$ or $\langle 10-10\rangle$ to $<0001>$. Significant grain refinement occurred in the NZ compared with AM60 BM, while grain growth with a certain degree occurred in the TMAZ of RS compared with AZ31 BM.

2 The grain size of AZ31 in NZ was obviously smaller than that of AM60. There was a higher percentage of LAGBs and a lower recrystallization degree in AZ31 side compared with AM60 side, especially for the TMAZ in AZ31 side. The discrepancies of the grain size distribution, recrystallization and LAGBs in joint were the results of the difference of initial state of two BMs and the inhomogeneous temperature distribution in joint.

3 The texture intensity of each micro-area in NZ showed an increase compared with BMs, while weaker texture intensity was presented in TMAZs. The texture from AS to RS in NZ presented a characteristic of symmetric distribution.

4 The tensile properties of joint among the three directions increased in the order: $\mathrm{TD}, 45^{\circ}$ and $\mathrm{WD}$, which can be explained by the difference of SF fluctuation for basal slip and extension twinning in various regions. Furthermore, the discrepancies of fracture behavior in the samples of $\mathrm{TD}$ and $45^{\circ}$ directions depended on the fluctuations of grain size and texture in joint.

Acknowledgements This work was financially supported by the National Key Research and Development Plan (No. 2016YFB0301104) and the National Natural Science Foundation of China (Nos. 51671041, 51531002, U1764253).

\section{References}

[1] J.L. Zhang, X. Chen, D.B. Xia, G.S. Huang, A.T. Tang, B. Jiang, F.S. Pan, Mater. Sci. Eng. A 778, 382 (2020)

[2] K. Singh, G. Singh, H. Singh, J. Magnes. Alloys 6, 399 (2018)

[3] J.L. Zhang, G.S. Huang, S.S. Liu, Y.L. Xie, G.G. Wang, B. Jiang, A.T. Tang, F.S. Pan, Mater. Sci. Eng. A 759, 479 (2019)

[4] A.H. Feng, Z.Y. Ma, Acta Mater. 57, 4248 (2009)

[5] P.K. Sahu, S. Pal, J. Magnes. Alloys 3, 36 (2015)

[6] P. Xue, X.X. Zhang, L.H. Wu, Z.Y. Ma, Acta. Metall. Sin. 52, 1222 (2016). (in Chinese) 
[7] X.H. Wang, K.S. Wang, Mater. Sci. Eng. A 431, 114 (2006)

[8] K. Singh, G. Singh, H. Singh, J. Magnes. Alloys 6, 292 (2018)

[9] S.H.C. Park, Y.S. Sato, Scr. Mater. 49, 161 (2003)

[10] Z. Liu, R.L. Xin, D.J. Liu, X.G. Shu, Q. Liu, Mater. Sci. Eng. A 658, 185 (2016)

[11] R.L. Xin, D.J. Liu, X.G. Shu, B. Li, X.F. Yang, Q. Liu, J. Alloys Compd. 670, 64 (2016)

[12] R.L. Xin, X. Zheng, Z. Liu, D.J. Liu, R.S. Qiu, Z.Y. Li, Q. Liu, J. Alloys Compd. 659, 51 (2016)

[13] C. Luo, X. Li, D. Song, N. Zhou, Y. Li, W. Qi, Mater. Sci. Eng. A 664, 103 (2016)

[14] D.J. Liu, R.L. Xin, X. Zheng, Z. Zhou, Q. Liu, Mater. Sci. Eng. A 561, 419 (2013)

[15] D. Liu, H. Nishio, K. Nakata, Mater. Des. 32, 4818 (2011)

[16] Z. Liu, R.L. Xin, X. Wu, D.J. Liu, Q. Liu, Mater. Sci. Eng. A 712, 493 (2018)

[17] W.K. Wang, W.C. Zhang, W.Z. Chen, J.L. Yang, L.X. Zhang, E.D. Wang, Mater. Sci. Eng. A 703, 17 (2017)

[18] Q. Shang, D.R. Ni, P. Xue, B.L. Xiao, Z.Y. Ma, Mater. Charact. 128, $14(2017)$

[19] A. Steuwer, M. Dumont, J. Altenkirch, S. Birosca, A. Deschamps, P.B. Prangnell, P.J. Withers, Acta Mater. 59, 3002 (2011)

[20] Z.Y. Ma, Q. Shang, D.R. Ni, B.L. Xiao, Acta Metall. Sin. 54, 1597 (2018). (in Chinese)

[21] R.L. Xin, B. Li, A.L. Liao, Z. Zhou, Q. Liu, Metall. Mater. Trans. A 43, 2500 (2012)
[22] U.F.H.R. Suhuddin, S. Mironov, Y.S. Sato, H. Kokawa, C.W. Lee, Acta Mater. 57, 5406 (2009)

[23] Y.M. Hwang, Z.W. Kang, Y.C. Chiou, H.H. Hsu, Int. J. Mach. Tools Manuf. 48, 778 (2008)

[24] H. Watanabe, H. Tsutsui, T. Mukai, H. Ishikawa, Y. Okanda, M. Kohzu, K. Higashi, Mater. Trans. 42, 1200 (2001)

[25] W.F. Xu, Y.X. Luo, M.W. Fu, Mate. Charact. 138, 48 (2018)

[26] W. Tang, X. Guo, J.C. Mcclure, L.E. Murr, J. Mater. Process. Manuf. 7, 163 (1998)

[27] M. Calcagnotto, D. Ponge, E. Demir, D. Raabe, Mater. Sci. Eng. A 527, 2738 (2010)

[28] Q. Shang, D.R. Ni, P. Xue, B.L. Xiao, Z.Y. Ma, Mater. Sci. Eng. A 707, 426 (2017)

[29] Y. Yao, C.M. Liu, Y.C. Wan, S.L. Yu, Y.H. Gao, S.N. Jiang, Mater. Charact. 161, 110 (2020)

[30] G.G. Wang, G.S. Huang, X. Chen, Q.Y. Deng, A.T. Tang, B. Jiang, F.S. Pan, Mater. Sci. Eng. A 707, 46 (2017)

[31] R.H. Li, F.S. Pan, B. Jiang, Q.S. Yang, A.T. Tang, Mater. Des. 46, $922(2013)$

[32] G. Liu, Z.D. Ma, G.B. Wei, T.C. Xu, X. Zhang, Y. Yang, W.D. Xie, X.D. Peng, J. Mater. Process. Technol. 267, 393 (2019)

[33] A. Raja, V. Pancholi, J. Mater. Process. Technol. 248, 8 (2017)

[34] D.J. Liu, R.L. Xin, L.Y. Sun, Z. Zhou, Q. Liu, Mater. Sci. Eng. A 576, 207 (2013)

[35] S. Mironov, T. Onuma, Y.S. Sato, S. Yoneyama, H. Kokawa, Mater. Sci. Eng. A 679, 272 (2017) 